\title{
Stabilization of Nanocrystalline Alloys via Grain Boundary Segregation: A Diffuse Interface Model
}

\author{
Fadi Abdeljawad ${ }^{\mathrm{a}, *}$, Stephen M. Foiles ${ }^{\mathrm{a}}$ \\ ${ }^{a}$ Computational Materials and Data Science, \\ Sandia National Laboratories, Albuquerque, New Mexico, 87185, USA
}

\begin{abstract}
Recent experimental and theoretical findings suggest that nanocrystalline binary alloys can be stabilized against interface-driven homogenization processes via grain boundary (GB) solute segregation mechanism. However, a detailed understanding of this process requires detangling the thermodynamic aspect, GB energy, from the kinetic one, GB mobility. In this work, we present a diffuse-interface model of GB segregation in binary metallic alloys that is capable of accounting for bulk thermodynamics, interfacial energies, and the interaction of alloying elements with GBs. In addition, the model presented herein extends current treatments by independently treating solute-solute interactions within both the bulk grain and GB regions, allowing for deviations from dilute and ideal systems and the ability to account for phase separation processes occurring in conjunction with grain growth. Starting with the analytical treatment of one-dimensional (1D) systems, we investigate the dependence of the GB energy, and subsequently the driving force for grain growth, on the segregation model parameters. More specifically, classic GB segregation isotherms are recovered in the limit of 1D infinite grains. Simulation results of two-dimensional systems reveal regimes of increased thermal stability, and highlight the importance of the thermodynamic model parameters of both bulk grain and GBs on grain growth processes. In broader terms, our modeling approach provides further avenues to explore GB solute segregation and its role in stabilizing polycrystalline aggregates.
\end{abstract}

Keywords: Grain boundary segregation, Grain growth, Phase field model,

\footnotetext{
${ }^{*}$ Corresponding author. e-mail: fabdelj@sandia.gov; Tel.: (505)845-9080; fax: (505) 844-9781. 
Thermodynamic stability, Nanocrystalline materials

\section{Introduction}

Owing to their nanometric characteristic feature size, nanocrystalline materials (NCs) exhibit a unique combination of properties [1-4] that render them an attractive choice in applications ranging from industrial and biomedical to optical and nanoelectronics [5-7]. In conventional microstructural materials, only a small fraction of atoms is spatially located at or in the immediate vicinity of grain boundaries (GBs). On the other extreme, NCs are characterized by a large volume fraction of GBs, with many atoms residing near interfaces $[1,6,8]$. This interfacial contribution is considered an Achilles' heel for any potential large scale commercialization as NCs are highly unstable against grain growth and coarsening processes even at low service temperatures [9-13]. These interface-driven processes lead to reduction in the total free energy, $\Delta G$, via the decrease in the total interfacial area and/or the reduction in interfacial energy due to changes in the geometrical attributes of the interface, i.e., $\Delta G \sim \Delta(\gamma A)$ [14]. The development of $\mathrm{NC}$ alloys with increased stability against grain growth is necessary to increase their competitiveness viz-à-viz other materials systems, especially in applications with harsh operating environments and elevated temperatures.

In the case of thermally driven grain growth and in accordance with the Gibbs-Thomson equation, the normal velocity, $\mathcal{V}_{n}$, at any point on the GB is given by $[14,15]$

$$
\mathcal{V}_{n}=M_{g b} P_{o}=M_{g b} \gamma_{g b} \mathcal{K},
$$

where $M_{g b}$ is the boundary mobility and $P_{o}$ is the driving force for grain growth, $\gamma_{g b}$ is the GB energy, and $\mathcal{K}$ is the local mean curvature. Broadly speaking, there are two main routes to stabilizing NCs against grain growth $[9$, $16,17]$. The first is the kinetic approach in which GB mobility is hindered via solute drag [18-20], or by pinning mechanisms due to a dispersion of closely spaced second-phase particles, commonly known as Zener pinning [21, 22]. On the other hand, thermodynamic stabilization occurs via the introduction of alloying elements into a polycrystalline material, which preferentially segregate to GBs; a process that results in reduction in the GB energy and thus the driving force for grain growth [16]. In fact, the reduction of interfacial energy via adsorption of solute atoms/active agents is commonly utilized 
in the study of fluid-fluid interfaces [23, 24]. Indeed, the Gibbs adsorption isotherm relates the surface density of solute at the interface (excess solute), $\Gamma$, to changes in the interfacial energy, $\gamma$, with respect to the solute chemical potential, $\mu$. This is quantified by [25]

$$
\Gamma=-\left(\frac{\partial \gamma}{\partial \mu}\right)_{P, T}
$$

where the partial differentiation is evaluated at constant pressure $P$ and temperature $T$. An alloying element that results in a reduction in GB energy via increasing its chemical potential will ultimately accumulate at the GB [25]. Classical adsorption (segregation) isotherms relate the solute concentration at the interface to the one in the bulk material far from interfaces. The so-called Langmuir-McLean isotherm [26] is considered the first treatment of equilibrium segregation at solid interfaces and it assumes that GB positions are substitutional sites, which are equally available for segregation [25, 27]. On the other hand, the Guttmann isotherm [28] deals with non-ideal multicomponent systems and it accounts for various types of solute-solute interactions at the GBs. The Fowler-Guggenheim isotherm [29] is considered a special case of the Guttmann model that applies to non-ideal binary metallic alloys [25].

Within the context of polycrystalline materials, Weissmüller [30] studied the thermal stability of nanostructures and the interaction of alloying elements with topological defects. Assuming a dilute system and utilizing the McLean isotherm, Weissmüller derived an analytical expression for the reduction in GB energy in terms of the GB heat of segregation [30]. Experimentally, several studies on binary NCs that are based on $\mathrm{Ni}$ [31-33], $\mathrm{Cu}[10,34], \mathrm{Fe}[35,36], \mathrm{W}[37]$, and $\mathrm{Pd}[38]$ have been conducted to examine grain growth and thermal stability in the presence of GB solute segregation. In all of the aforementioned experimental work, it was observed that the proper choice of the alloying element, resulting in a positive heat of segregation, leads to nanostructures with increased thermal stability. Moreover, it was suggested that the reduction in GB energy by virtue of solute segregation to GBs is the underlying mechanism for the observed stabilization. Others propose that the thermodynamic and kinetic routes are deeply intertwined and both act simultaneously, where their degree of influence is dictated by the segregation and diffusion of solute atoms to GBs [7, 10, 34]. Recently, stability maps have been constructed for various combinations of binary ele- 
ments to infer information on the energetics of GB segregation and thermal stability of these alloys [39-41].

Analytical and thermodynamic treatments of binary NCs have been developed to relate GB energy to segregation energetics [17, 40, 42]. Typically, these studies utilize the regular solution approximation in combination with the Gibbs adsorption equation to derive simple relations for the dependence of GB energy on thermodynamic parameters. Atomistic studies provide valuable insights on the preferential GB sites for segregation and anisotropic effects but are unable to attain diffusive time scales associated with homogenization processes [32, 43-46]. At the mesoscale, modeling efforts are focused on investigating solute drag in dilute systems and have been successfully mapped onto classic treatments of solute drag [47-51]. Motivated by the work of Grönhagen and Ågren [48], Kim and Park [49], assuming an ideal solution with bulk concentration $c=0.01$, examined the shrinking kinetics of an isolated grain embedded in a matrix grain in the presence of GB segregation. In their study, the McLean isotherm was utilized to investigate the role of atomic diffusivity on the evolution of the circular grain and concentration profile. Recently, Heo et al. [51] extended the framework detailed in $[47,48]$ to account for strain-induced GB segregation and the resulting solute drag effects. While these treatments provide useful insights on the role of GB segregation on grain growth, they only examine the kinetic aspect of GB segregation, i.e., solute drag, and do not account for solute-solute interactions within GBs or deviations from ideal and dilute systems [47-51].

In this work, we develop a theoretical and computational phase field framework for the microstructural evolution of binary NCs with the aim of quantifying the thermodynamic aspects of GB segregation. We investigate, in a parametric study, the role of various thermodynamic parameters on grain growth dynamics. Starting with one dimensional systems, the equilibrium properties are derived and the dependences of the GB energy and width on model parameters are described. Next, we survey through simulations of two-dimensional systems the phase-space of model parameters and reveal regimes with increased thermodynamic stability. The rest of the manuscript is organized as follows: The theoretical modeling framework is presented in Section 2, while the equilibrium properties and the resulting GB segregation isotherms are presented in Section 3. Simulation results of various two-dimensional systems are presented and discussed in Section 4. Finally, this manuscript is concluded by Section 5, which provides a summary and discusses our future work on this model. 


\section{Theoretical Framework}

The starting point of our phase field model is the introduction of structural order parameters (OPs) $\left\{\phi_{i}(\mathbf{r}, t), i=1, \cdots, n_{\phi}\right\}$ that describe grains in an alloy with various crystallographic orientations. Here, $\mathbf{r}, t, n_{\phi}$ denote the position vector, time and number of OPs needed to resolve the microstructure, respectively. In this study, we focus on binary metallic alloys of substitutional elements A (host) and B (solute), where a conserved solute concentration field $c=c(\mathbf{r}, t)$ is defined throughout the alloy. Next, we propose the following for the total free energy functional, $\mathcal{F}_{t o t}$, of a polycrystalline binary alloy

$$
\begin{aligned}
\mathcal{F}_{\text {tot }} & =\int d \mathbf{r} f_{\text {tot }}\left(c, T, \phi_{i}\right) \\
& =\int d \mathbf{r}\left[f_{\text {mix }}(c, T)+W_{\phi} f_{\text {grain }}\left(\phi_{i}\right)+\sum_{i}^{n_{\phi}} \frac{\epsilon_{i}^{2}}{2}\left|\nabla \phi_{i}\right|^{2}-f_{\text {inter }}\left(c, \phi_{i}\right)\right]
\end{aligned}
$$

where $f_{\text {mix }}$ is the homogeneous free energy density, $f_{\text {grain }}\left(\phi_{i}\right)=f_{\text {grain }}\left(\left\{\phi_{i}(\mathbf{r}, t), i=\right.\right.$ $\left.\left.1, \cdots, n_{\phi}\right\}\right)$ is the free energy density of the grain microstructure and $W_{\phi}$ is a parameter that sets the energy scale. The third term on the right hand side of Eq. (3) describes GB energy, where under the isotropy assumption $\epsilon_{i}=\epsilon$. This assumption will be invoked for the remainder of this study, but could be generalized in future work. Also, one could add to $f_{\text {tot }}$ an additional term $f_{\text {phase }}=\left(\kappa^{2} / 2\right)|\nabla c|^{2}$ to account for the interfacial energy due to compositional domains in phase-separating systems, where $\kappa$ is a model parameter that sets the energy of phase boundaries between A-rich and B-rich domains. $f_{\text {inter }}\left(c, \phi_{i}\right)=f_{\text {inter }}\left(c,\left\{\phi_{i}(\mathbf{r}, t), i=1, \cdots, n_{\phi}\right\}\right)$ is an interaction term that couples the solute concentration field, $c(\mathbf{r}, t)$, to the grain microstructure and whose explicit form will be discussed further below. Under the regular solution approximation, the homogeneous free energy density is written as

$$
f_{\text {mix }}=G_{B} c+G_{A}(1-c)+\frac{R T}{V_{m}}[c \ln c+(1-c) \ln (1-c)]+\Omega c(1-c),
$$

where $G_{B}$ and $G_{A}$ are the free energy densities in pure states, $R, T, V_{m}$, and $\Omega$ are the ideal gas constant, temperature, molar volume (assumed the same for A and B elements), and enthalpic term coefficient (heat of mixing), respectively. The OPs are conveniently chosen such that the equilibrium values within a grain $\left\{\phi_{i}(\mathbf{r}, t)=1, \phi_{j \neq i}(\mathbf{r}, t)=0, i, j=1, \cdots, n_{\phi}\right\}$ and 
$\left\{\phi_{i}(\mathbf{r}, t) \in(0,1), i=1, \cdots, n_{\phi}\right\}$ define GB regions. Motivated by the work of one of the authors (FA) on grain growth and coarsening in solid oxide fuel cells [52] and the treatment of solid-state sintering by Wang [53], we adopt the following form for the free energy of a grain microstructure

$$
f_{\text {grain }}=\frac{4}{3}\left[1-4 \sum_{i=1}^{n_{\phi}} \phi_{i}^{3}+3\left(\sum_{i=1}^{n_{\phi}} \phi_{i}^{2}\right)^{2}\right],
$$

where again the minima of $f_{\text {grain }}$ are located at $\left\{\phi_{i}\right\}=\{1,0, \cdots, 0\}=\cdots=$ $\{0,0, \cdots, 1\}$. Therefore, within this mesoscale treatment the order parameters, $\phi_{i}$, attain their equilibrium values within the bulk grains and vary smoothly but sharply across GBs.

For the interaction term, we adopt the following form

$$
f_{\text {inter }}\left(c, \phi_{i}\right)=\left(\xi_{0}+\xi_{1} c+\xi_{2} c^{2}\right) \psi\left(\phi_{i}\right),
$$

where the multiplicative decomposition assumes a quadratic dependence on the concentration. $\xi_{0}, \xi_{1}$ and $\xi_{2}$ are model parameters and the function $\psi\left(\phi_{i}\right)$ is a smoothly but rapidly varying function that takes the value of one within GBs and zero elsewhere and is used to locate the interaction energy to GB regions only. In this work, we let $\psi\left(\phi_{i}\right)=f_{\text {grain }}\left(\phi_{i}\right)$, where inspection of Eq. (5) reveals that $f_{\text {grain }}=0, \forall \phi_{i}=1, \phi_{j \neq i}=0$, and $f_{\text {grain }} \neq 0, \forall \phi_{i}(\mathbf{r}, t) \in$ $(0,1), i, j=1, \cdots, n_{\phi}$. Furthermore, at the center of the GB region, where $\psi\left(\phi_{i}\right)=1$, one can write for the GB regions an effective chemical component of the free energy, $f_{m i x}^{e f f}$, that takes the following form

$$
\begin{aligned}
f_{\text {mix }}^{\text {eff }} & =\left(G_{A}-\xi_{0}\right)+\left(G_{B}-G_{A}+\Omega-\xi_{1}\right) c \\
& -\left(\Omega+\xi_{2}\right) c^{2}+\frac{R T}{V_{m}}[c \ln c+(1-c) \ln (1-c)] .
\end{aligned}
$$

Therefore, the interaction term $f_{\text {inter }}$ effectively assigns the GB regions thermodynamic properties that are distinct from the bulk grains, where the model parameters $\xi_{0}, \xi_{1}$ and $\xi_{2}$ set the deviation from bulk grain properties. Rearrangement of Eq. (7) to a form similar to the one in Eq. (4) yields the following for the effective thermodynamic properties of the GB regions

$$
\begin{aligned}
G_{A}^{g b} & =G_{A}-\xi_{0}, \\
G_{B}^{g b} & =G_{B}-\left(\xi_{0}+\xi_{1}+\xi_{2}\right), \\
\Omega^{g b} & =\Omega+\xi_{2},
\end{aligned}
$$


where $G_{A}^{g b}$ and $G_{B}^{g b}$ are the free energy density of pure states and $\Omega^{g b}$ sets the enthalpic term all within the GB regions. In this work, the parameter $\xi_{0}$, which merely offsets the effective GB free energy curves, will be assumed zero since it does not contribute to the dynamics, i.e., the chemical potential for the concentration field is obtained via the variational derivative of the energy functional in Eq. (3). In the dilute limit and for ideal systems, i.e., $\Omega=0$, GB segregation is accounted for by the linear term in $f_{\text {inter }}$, where $\xi_{1}$ sets the degree of GB segregation. On the other hand, solute-solute interactions within GBs are captured through the quadratic term in $f_{\text {inter }}$ and $\xi_{2}$ sets the strength of this interaction. It is worth mentioning that current mesoscale models for GB segregation in binary alloys consider ideal systems with uniform segregation and no solute-solute interactions within GBs [47-50]; an approach only valid in the dilute limit [25].

The dynamics of binary alloys follow from the minimization of $\mathcal{F}_{t o t}$. Within the Ginzburg-Landau formalism and with the aid of variational principles, the governing equations for the evolution of the concentration field $c(\mathbf{r}, t)$ and $\operatorname{OPs}\left\{\phi_{i}(\mathbf{r}, t), i=1, \cdots, n_{\phi}\right\}$ for $(\mathbf{r}, t) \in V \times\left[0, t_{\text {final }}\right] \subset \mathbb{R}^{3} \times \mathbb{R}$, where $V$ is the domain volume and $t_{\text {final }}$ is the simulation time, can be stated respectively as $[54,55]$

$$
\begin{aligned}
\frac{\partial c}{\partial t} & =\nabla \cdot\left[M_{c} \nabla\left(\frac{\delta \mathcal{F}_{t o t}}{\delta c}\right)\right], \\
\frac{\partial \phi_{i}}{\partial t} & =-L_{i} \frac{\delta \mathcal{F}_{t o t}}{\delta \phi_{i}}, i=1, \cdots, n_{\phi},
\end{aligned}
$$

where $M_{c}$ is the atomic mobility function and in general has the degenerate form $M_{c}=M_{o} Q\left(c, \phi_{i}\right)$, where $M_{o}$ is the intrinsic atomic mobility and $Q\left(c, \phi_{i}\right)$ is a function used to define inhomogeneities in diffusion pathways. The model parameters $L_{i}$, one for each of the $\phi_{i}$, are related to GB mobilities, where under the isotropy assumption $L_{i}=L$.

\section{Equilibrium Properties}

\subsection{GB segregation isotherm}

In this section, we examine the equilibrium properties of the phase field model in one-dimension (1D). This will elucidate the relationship between the model parameters and the interfacial properties. To this end, we consider a 1D bicrystal, where the first grain is described by the OP $\phi_{1}$ that extends 
spatially in the region $x \in\left(-\infty,-\delta_{G B} / 2\right)$. The second grain is tracked via the OP $\phi_{2}$ and is defined in the region $x \in\left(+\delta_{G B} / 2,+\infty\right)$. Note that within this diffuse-interface description, the $\mathrm{GB}$ is defined in the region $x \in$ $\left[-\delta_{G B} / 2,+\delta_{G B} / 2\right]$, where $\delta_{G B}$ is the GB width. Furthermore, due to symmetry the OPs are related through $\phi_{2}=1-\phi_{1}$ and only one phase field is required to describe this $1 \mathrm{D}$ system. Here, we let $\phi_{1}=\phi$ and the grain microstructure free energy density becomes $f_{\text {grain }}=16 \phi^{2}(1-\phi)^{2}$. At equilibrium, the stationary fields $c_{e}=c_{e}(\mathbf{r}, t \rightarrow \infty)$ and $\phi_{e}=\phi_{e}(\mathbf{r}, t \rightarrow \infty)$ obey the following relationships for the chemical potentials of $c, \mu_{c}$, and $\phi, \mu_{\phi}$

$$
\begin{array}{r}
\mu_{c}=\frac{\delta \mathcal{F}_{\text {tot }}}{\delta c}=\left.\frac{\partial f_{\text {mix }}}{\partial c}\right|_{c_{e}}-16\left(\xi_{1}+2 \xi_{2} c_{e}\right) \phi_{e}^{2}\left(1-\phi_{e}\right)^{2}=\text { const. } \\
\mu_{\phi}=\frac{\delta \mathcal{F}_{\text {tot }}}{\delta \phi}=\left.W_{\phi} \frac{\partial f_{\text {grain }}}{\partial \phi}\right|_{\phi_{e}}-\epsilon^{2} \nabla^{2} \phi_{e}-\left.\left(\xi_{1} c_{e}+\xi_{2} c_{e}^{2}\right) \frac{\partial f_{\text {grain }}}{\partial \phi}\right|_{\phi_{e}}=0
\end{array}
$$

where $\partial f_{\text {mix }} / \partial c=G_{B}-G_{A}+\left(R T / V_{m}\right) \ln (c /(1-c))+\Omega(1-2 c)$.

Once the homogeneous free energy of the bulk grain and GB regions are established, the equilibrium concentration within the GB is obtained as follows: A tangent to the bulk grain free energy curve is drawn at the bulk concentration. Then, the equilibrium GB concentration is obtained by establishing a tangent to the GB free energy curve that is parallel to the one drawn for the bulk grain [25]. This is illustrated schematically in Fig. 1(a), where the dashed lines are tangents to $f_{m i x}$ curves. The vertical dashed lines are drawn to guide the eye to the tangent points, which represent the equilibrium far-field bulk, $c_{\infty}$, and $\mathrm{GB}, c_{g b}$, concentrations. We note that in Fig. 1(a), only the chemical contribution of the free energy is plotted, i.e., $f_{m i x}$, while the GBs have an additional energy contribution due to the third term on the right hand side of Eq. (3). The pictorial representation of the equilibrium GB concentration can be cast mathematically by letting $c_{\infty}=c_{e}(\mathbf{r} \rightarrow \pm \infty)$ within the bulk grain $\left(\phi_{e}=0\right.$ or 1) far from the GB. Then, Eq. (11) is applied to both the bulk grain and $\mathrm{GB}$ regions to yield

$$
\left.\frac{\partial f_{m i x}}{\partial c}\right|_{c_{e}}-\left.\frac{\partial f_{m i x}}{\partial c}\right|_{c_{\infty}}=16\left(\xi_{1}+2 \xi_{2} c_{e}\right) \phi_{e}^{2}\left(1-\phi_{e}\right)^{2},
$$

where we note that the spatial dependence of the concentration field within and in the vicinity of the GB is captured via $c_{e}(\mathbf{r})$. Next, Eq. (13) is solved 


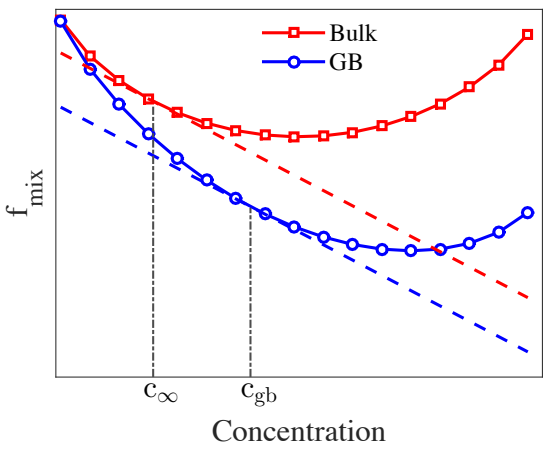

(a)

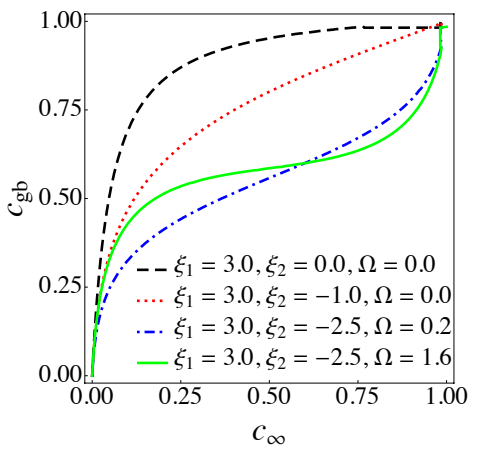

(b)

Figure 1: (Color online). (a) Schematic representation of the chemical component of the free energy, $f_{m i x}$, for both the bulk grain and GB regions. The equilibrium GB concentration is constructed by establishing a tangent to the GB free energy curve, dashed blue line, that is parallel to the tangent line for the bulk grain free energy curve, dashed red line, at the far-field bulk concentration. The vertical dashed lines are drawn to guide the eye to the tangent points. (b) With the aid of Eq. (15), plots of the GB segregation isotherm for various values of $\xi_{1}, \xi_{2}$ and $\Omega$. Here, it was assumed that $R T / V_{m}=1.0$ and $\Delta H_{\text {seg }}$ is evaluated at the center of the GB, where $\phi_{e}=0.5$ and $c_{g b}=c_{e}\left(\mathbf{r} \mid \phi_{e}(\mathbf{r})=0.5\right)$. Note that the McLean isotherm is recovered when $\xi_{2}=\Omega=0$ (dashed black line).

with the aid of Eq. (4) for $f_{m i x}$ to yield a GB segregation isotherm, which relates the equilibrium concentration at a position $\mathbf{r}$ from the GB to the far-field bulk grain concentration, $c_{\infty}$, that takes the following form

$$
\frac{c_{e}(\mathbf{r})}{1-c_{e}(\mathbf{r})}=\frac{c_{\infty}}{1-c_{\infty}} \exp \left[\frac{\Delta H_{s e g}}{\left(R T / V_{m}\right)}\right]
$$

where $\Delta H_{\text {seg }}$ is the GB segregation energy and is written as follows

$$
\Delta H_{\text {seg }}=16 \xi_{1} \phi_{e}^{2}\left(1-\phi_{e}\right)^{2}+32 \xi_{2} \phi_{e}^{2}\left(1-\phi_{e}\right)^{2} c_{e}+2 \Omega\left(c_{e}-c_{\infty}\right) .
$$

The resulting form for the segregation energy warrants some attention. The first term on right hand side of Eq. (15) is concentration-independent, where the model parameter $\xi_{1}$ sets the contribution to $\Delta H_{\text {seg. }}$. This term can be mapped onto the classic Langmuir-McLean isotherm [27], where it is assumed that GB positions are equally available for segregation and is applied to binary systems dilute enough to exhibit ideal behavior [25]. The second term on the right hand side of Eq. (15) is concentration-dependent, and there- 
fore accounts for solute-solute interactions within the GB region. The resulting form for $\Delta H_{\text {seg }}$ is similar to the Fowler-Guggenheim isotherm [25, 29], where $\xi_{2}<0$ treats repulsive B-B solute atom interactions and leads to lowering the segregation energy as the GB concentration increases. On the other hand, attractive B-B interactions are obtained by setting $\xi_{2}>0$. In this case, the extent of segregation is enhanced with increasing GB concentration [25, 27]. Finally, our treatment of binary alloys and the resulting segregation energy, i.e., Eq. (15), is similar in form to recent analytical treatments, where the segregation energy is non-uniform and depends on both the concentration in the bulk grain and GB regions [17, 40].

To demonstrate the capability of the current framework to model a wide variety of segregation behaviors, we calculated segregation isotherms at the center of the GB, where $\phi_{e}=0.5$ and $c_{g b}=c_{e}\left(\mathbf{r} \mid \phi_{e}(\mathbf{r})=0.5\right)$ is the concentration at the center of the GB region. Plots of the resulting segregation isotherm for various combinations of $\xi_{1}, \xi_{2}$ and $\Omega$ are displayed in Fig. 1(b). In the dilute limit, i.e., $c_{\infty} \lesssim 0.03$, the plotted isotherms yield similar GB concentrations but deviate considerably at larger $c_{\infty}$ values. For a specific alloy system, the values of $\xi_{1}$ and $\xi_{2}$ would be chosen to match segregation isotherms obtained experimentally or via atomistic simulations.

One last note regarding the resulting segregation isotherm. Anisotropy effects in the GB segregation can be incorporated by allowing both $\xi_{1}$ and $\xi_{2}$ to depend on the GB type and character. For example, the dependence on GB inclination can be incorporated by simply letting $\xi_{1}=z_{1}(\theta)$ and $\xi_{2}=z_{2}(\theta)$, where $\theta$ is the inclination angle of the GB plane with respect to the global reference frame.

\subsection{GB energy and width}

With the aid of the 1D geometry described at the beginning of Section 3, we seek to examine the equilibrium properties of the phase field, $\phi_{e}$. As shown in Appendix A, the equilibrium profile of $\phi_{e}$ satisfies

$$
\begin{aligned}
& \frac{\epsilon^{2}}{2}\left(\frac{d \phi_{e}}{d x}\right)^{2}=16 W_{\phi} \phi_{e}^{2}\left(1-\phi_{e}\right)^{2}-16\left(\xi_{1} c_{e}+\xi_{2} c_{e}^{2}\right) \phi_{e}^{2}\left(1-\phi_{e}\right)^{2} \\
& +\frac{R T}{V_{m}}\left[c_{e} \ln \left(\frac{c_{e}}{c_{\infty}}\right)+\left(1-c_{e}\right) \ln \left(\frac{1-c_{e}}{1-c_{\infty}}\right)\right]-\Omega\left(c_{\infty}-c_{e}\right)^{2}
\end{aligned}
$$

where it is evident that the equilibrium concentration, $c_{e}$, and phase field, $\phi_{e}$, are coupled through Eqs. (13) and (16). Following the treatment of solute 
trapping during solidification by Wheeler et al. [56], it is shown in Appendix $\mathrm{B}$ that the concentration-dependent GB energy, $\gamma_{g b}$, is written as

$$
\gamma_{g b}=\epsilon^{2} \int_{-\infty}^{+\infty}\left(\frac{d \phi_{e}}{d x}\right)^{2} d x=\epsilon^{2} \int_{0}^{1} \frac{d \phi_{e}}{d x} d \phi_{e}
$$

Furthermore, the distance by which the phase field, $\phi_{e}$, changes from $\phi_{\text {in }}$ to $\phi_{\text {out }}$, where $\phi_{\text {in }}$ and $\phi_{\text {out }}$ are lower and upper bounds on the phase field, $\phi_{e}$, is used as a measure of GB width, $\delta_{g b}$. Here, we set $\left(\phi_{\text {in }}, \phi_{\text {out }}\right)=(0.1,0.9)[50]$ and the GB width is given by

$$
\delta_{g b} \simeq \int_{\phi_{\text {in }}}^{\phi_{\text {out }}} \frac{d x}{d \phi_{e}} d \phi_{e} .
$$

In the special case of no GB segregation, where $\xi_{1}=\xi_{2}=0$ and $c_{e}=c_{\infty}$ everywhere, and following the treatment of interfaces by Cahn and Hilliard [54], Eq. (12) can be solved analytically for $\phi_{e}$, which admits a traveling wave solution

$$
\phi_{e}(x)=\frac{1}{2}\left[1-\tanh \left(\frac{2 \sqrt{2 W_{\phi}} x}{\epsilon}\right)\right] .
$$

The analytical solution for $\phi_{e}$ in combination with Eqs. (17) and (18) yields the following for the GB energy, $\gamma_{g b}$, and width, $\delta_{g b}$, in the absence of GB solute segregation

$$
\begin{aligned}
\gamma_{g b} & =\frac{2 \sqrt{2}}{3} \epsilon \sqrt{W_{\phi}} \\
\delta_{g b} & \simeq \frac{1.1}{\sqrt{2}} \frac{\epsilon}{\sqrt{W_{\phi}}}
\end{aligned}
$$

To gain a better understanding of the role of GB segregation on the equilibrium properties, we numerically evaluated the GB energy and width for various values of the segregation model parameters $\xi_{1}$ and $\xi_{2}$. First, we Taylor expand the exponential part of Eq. (14) up to $\mathcal{O}\left(c_{e}^{3}\right)$ and collect the terms to form a polynomial of the form

$$
\frac{\beta^{2}}{2} c_{e}^{3}-\beta c_{e}^{2}+(1+\alpha) c_{e}-\alpha=0,
$$




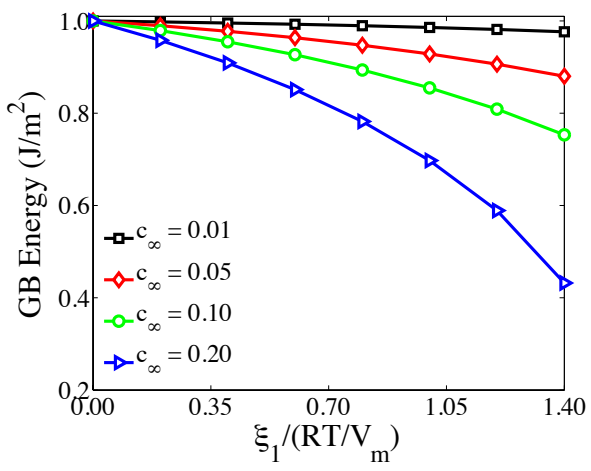

(a)

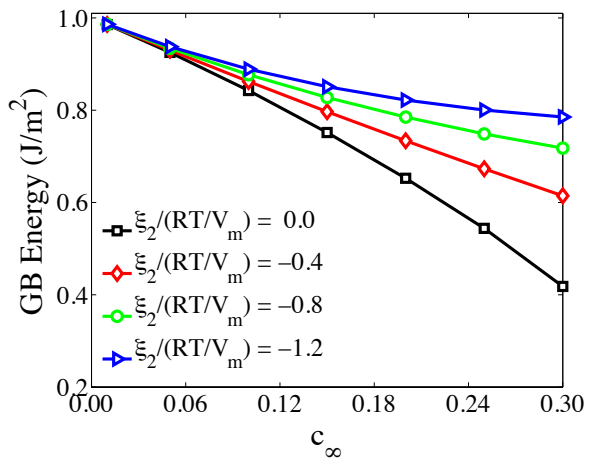

(c)

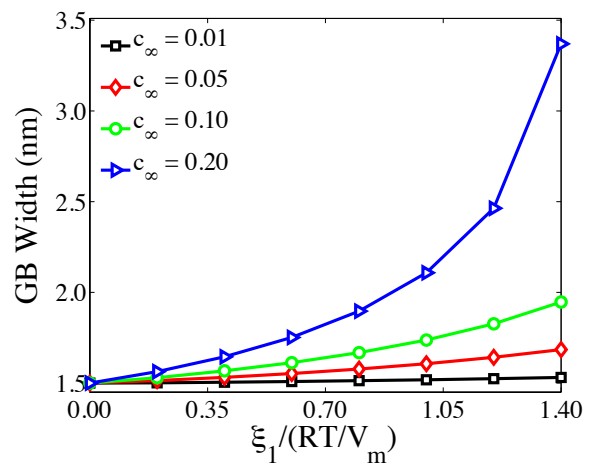

(b)

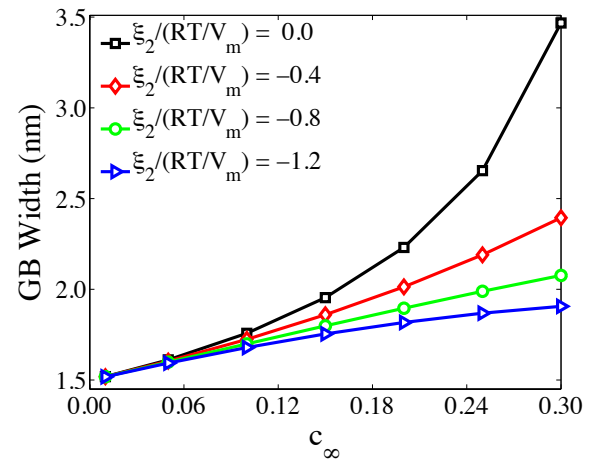

(d)

Figure 2: (Color online). Plots of GB energy [(a) and (c)] and width [(b) and (d)] as a function of GB segregation model parameters. (a) and (b): The role of $\xi_{1}$ for a system with $\xi_{2}=\Omega=0$ at various values of the far-field bulk concentration, $c_{\infty}$. (c) and (d): The role of $c_{\infty}$ for a system with $\xi_{1} /\left(R T / V_{m}\right)=1.0$ and $\Omega /\left(R T / V_{m}\right)=0.75$ at various values of the GB solute-solute interaction parameter, $\xi_{2}$. Here, it was assumed that $T=650^{\circ} \mathrm{C}$, $V_{m}=7 \times 10^{-6} \mathrm{~m}^{3} \mathrm{~mol}^{-1}$ and $\left(\gamma_{g b}, \delta_{g b}\right)=\left(1.0 \mathrm{~J} \mathrm{~m}^{-2}, 1.5 \mathrm{~nm}\right)$ initially in the absence of GB segregation.

where $\alpha=\left(c_{\infty} /\left(1-c_{\infty}\right)\right) \exp \left[\left(16 \xi_{1} \phi_{e}^{2}\left(1-\phi_{e}\right)^{2}-2 \Omega c_{\infty}\right) /\left(R T / V_{m}\right)\right]$ and $\beta=$ $\left(32 \xi_{2} \phi_{e}^{2}\left(1-\phi_{e}\right)^{2}+2 \Omega\right) /\left(R T / V_{m}\right)$. The real root $c^{*}=c_{e}(\alpha, \beta)$ is inserted in Eq. (16), which is then used for the numerical integration of Eqs. (17) and (18) to calculate the GB energy and width, respectively. These calculations were performed using Mathematica v9.0 [57] assuming $T=650^{\circ} \mathrm{C}$, $V_{m}=7 \times 10^{-6} \mathrm{~m}^{3} \mathrm{~mol}^{-1}$ and $\left(\gamma_{g b}, \delta_{g b}\right)=\left(1.0 \mathrm{~J} \mathrm{~m}^{-2}, 1.5 \mathrm{~nm}\right)$, which are representative of metallic systems in the absence of solute segregation [58]. The results of these calculations are displayed in Fig. 2, where panels (a) 
and (b) examine the dependence of GB energy and width, respectively, on the model parameter $\xi_{1}$, while setting $\xi_{2}=\Omega=0$, for various values of the far-field bulk concentration, $c_{\infty}$. It can be seen that increasing the value of $\xi_{1}$ leads to a reduction in GB energy and an increase in GB width. Also, larger values for the far-field concentration are more effective in reducing the GB energy. In the dilute limit, the decrease in GB energy with increasing $\xi_{1}$ is very modest and solute drag is expected to be the operating mechanism [49, 50]. Next, Figs. 2(c) and (d) display the dependence of the GB energy and width, respectively, on the far-field concentration for various values of the GB solute-solute interaction parameter, $\xi_{2}$. Here, it was assumed that $\xi_{1} /\left(R T / V_{m}\right)=1.0$ and $\Omega /\left(R T / V_{m}\right)=0.75$. It is evident that as the value of $\xi_{2}$ decreases below zero, the effect of solute-solute interactions becomes increasingly more dominant. This repulsive interaction, when $\xi_{2}<0$, yields less accumulation of solute at the GB, and therefore is less efficient in reducing the GB energy. At small values for the far-field concentration, $c_{\infty}$, solute-solute interactions are less significant and the segregation behavior is mainly driven by $\xi_{1}$.

\section{Results and Discussion}

In this section, we examine, in two-dimensions (2D), the microstructural evolution of various systems in response to GB segregation isotherms that are prescribed via the parameters $\xi_{1}$ and $\xi_{2}$. To this end, the evolutionary equations for $c(\mathbf{r}, t)$ and $\left\{\phi_{i}(\mathbf{r}, t), i=1, \cdots, n_{\phi}\right\}$ are made non-dimensional by introducing dimensionless space and time as $\tilde{\mathbf{r}}=\mathbf{r} / d_{o}$, and $\tilde{t}=t / \tau$, respectively, where $d_{o}$ and $\tau$ denote the characteristic length (pixel edge length) and time scales, respectively. For convenience, we introduce the non-dimensional quantities $\tilde{G}_{A}=G_{A} /\left(R T / V_{m}\right), \tilde{G}_{B}=G_{B} /\left(R T / V_{m}\right), \tilde{\Omega}=$ $\Omega /\left(R T / V_{m}\right), \tilde{\xi}_{1}=\xi_{1} /\left(R T / V_{m}\right)$ and $\tilde{\xi}_{2}=\xi_{2} /\left(R T / V_{m}\right), \tilde{W}_{\phi}=W_{\phi} /\left(R T / V_{m}\right)$ and $\tilde{\epsilon}^{2}=\epsilon^{2} /\left(d_{o}^{2}\left(R T / V_{m}\right)\right)$. Additionally, atomic mobility can be written as $M_{o} \simeq D_{o} /\left(\partial^{2} f_{\text {mix }} / \partial c^{2}\right)_{e q}$, where $D_{o}$ is the solute diffusivity. Inserting the above non-dimensional quantities into Eq. (9) yields the following for the characteristic time scale

$$
\tau=\frac{4 d_{o}^{2} V_{m}}{M_{o} R T} \simeq \frac{\tilde{\epsilon}^{2} d_{o}^{4}}{D_{o} \tilde{W}_{\phi} \delta_{g b}^{2}} .
$$

Furthermore, we introduce the non-dimensional boundary mobility $\tilde{L}=$ $\tau L\left(R T / V_{m}\right)$ in the governing equation, Eq. (10), for the non-conserved struc- 
tural OPs, $\phi_{i}$. The model parameters $L$ and $\epsilon$ are related to GB mobility, $M_{g b}$, and energy, $\gamma_{g b}$, through $[59,60]$

$$
M_{g b} \gamma_{g b}=L \epsilon^{2}=\frac{\tilde{L}}{\tau} \tilde{\epsilon}^{2} d_{o}^{2} \simeq \frac{\tilde{L} D_{o} \tilde{W}_{\phi} \delta_{g b}^{2}}{d_{o}^{2}},
$$

where Eq. (23) has been used in the last step. The resulting non-dimensional governing equations for $c(\mathbf{r}, t)$ and $\phi_{i}(\mathbf{r}, t)$ have the same form as their dimensional counterparts, i.e., Eqs. (9) and (10), where we assumed $Q\left(c, \phi_{i}\right)=1$, i.e., solute diffusivity is constant everywhere. The results reported in this work are therefore expressed temporally and spatially in terms of $\tau$ and $d_{o}$, respectively. For the remainder of this paper, we drop the tildes on all parameters for notational convenience.

Representative systems are discretized to produce a simulation box of $N_{x} \times N_{y}$ pixels, where the pixel size (edge length) is $\Delta x=\Delta y=1.0$ along the $\mathrm{x}$ and $\mathrm{y}$ directions, respectively. Model parameters were chosen such that the GB width is resolved by six grid points (pixels), which provides a good compromise between numerical accuracy and computational tractability [49]. The equations are solved using explicit Euler method for the time derivatives and central finite differencing with five-point stencils for spatial gradients. Periodic boundary conditions along all spatial dimensions for all fields are used.

\subsection{An oval racetrack grain geometry}

It is ideal in the study of GB motion to separate the GB energy contribution from the one due to interface curvature. Therefore, we start our exploration of the model by examining a $2 \mathrm{D}$ system that consists of a grain in the shape of an oval racetrack, or oblong, embedded in a larger matrix grain as shown in Fig. 3(a). According to Eq. (1), the driving force for grain growth is linearly proportional to the product of the interface energy and local mean curvature. The configuration depicted in Fig. 3(a) is conveniently chosen since it results in a controlled grain shrinkage, where the local curvature is kept constant during the evolution process. In this system, GB migration occurs via the inward horizontal motion of the semi-circular caps, while maintaining their local curvature, along the reference $\mathrm{x}$-axis. This evolution process is schematically shown in Fig. 3(b), where the time scales are such that $t_{1}<t_{2}<t_{3}$. Other variants of the configuration depicted in Fig. 3(a) have been used before in the study of GB motion [61, 62]. 


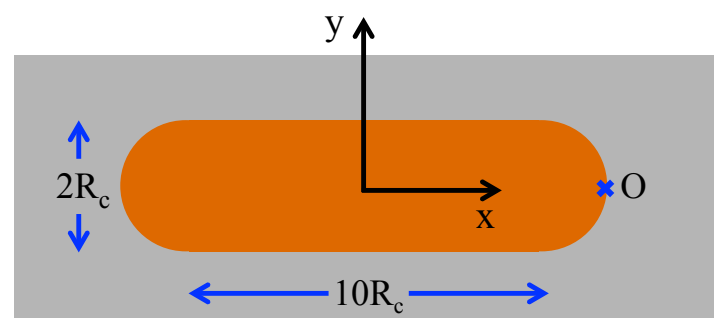

(a)

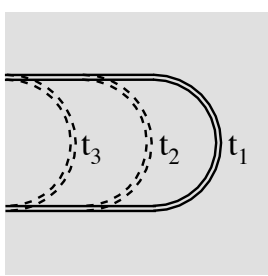

(b)

Figure 3: (Color online). A two-grain system that consists of an oval racetrack-shaped grain (orange) embedded in a larger matrix grain (grey). To quantify growth dynamics, we numerically track the spatial position along $\mathrm{x}$ and solute concentration of the GB point "O" (at the center of the GB), which is located at the apex of the right semi-circular cap of the oblong grain. (b) A schematic illustrating the temporal evolution of the right semi-circular cap of the oblong grain. Here the time scales are such that $t_{1}<t_{2}<t_{3}$.

In all the simulations presented in this section, we quantified GB migration by tracking the spatial position along $\mathrm{x}$ and solute concentration of a point, which we denote point $\mathrm{O}$, at the center of the GB that is positioned at the apex of the right semi-circular cap of the oblong grain. The point $\mathbf{O}$, which is shown in Fig. 3(a), corresponds to the GB center point, where $\phi(\mathbf{r}, t)=0.5$. This center point may not coincide with an actual numerical grid point (pixel) and in cases it did not, the closest grid point to the GB center was chosen and its concentration was recorded. Due to symmetry, only the right semi-circular boundary was considered in the results presented in this section. A system was constructed with $\left(N_{x}, N_{y}, R_{c}\right)=(450,175,25)$, where $R_{c}$ is the radius of the semi-circular caps. In the following simulations, we set $G_{A}=G_{B}=1.0$, $\left(W_{\phi}, \epsilon, L\right)=(0.4,1.9,1.0)$ and the energy scale $R T / V_{m} \simeq 6.7 \times 10^{8} \mathrm{~J} \mathrm{~m}^{-3}$, where $V_{m}=10.0 \times 10^{-6} \mathrm{~m}^{3} \mathrm{~mol}^{-1}$. Furthermore, we let the atomic diffusivity $D_{o} \sim 10^{-15} \mathrm{~m}^{2} \mathrm{~s}^{-1}$ [37], characteristic length scale $d_{o}=2.0 \mathrm{~nm}$ and use a numerical time step $\Delta t=0.0001$. These parameters correspond to an interfacial energy $\gamma_{g b} \sim 1.4 \mathrm{~J} \mathrm{~m}^{-2}$ initially in the absence of GB segregation. GB mobility can vary by orders of magnitude depending on several factors, such as temperature, and GB type and character [63-65]. In this work, GBs were assumed to be of the same type, where $M_{g b} \sim 10^{-15} \mathrm{~m}^{4} \mathrm{~J}^{-1} \mathrm{~s}^{-1}$ [51]. We note that the parameters chosen in this study correspond to a characteristic time $\tau \sim 1.0 \mathrm{~s}$. 


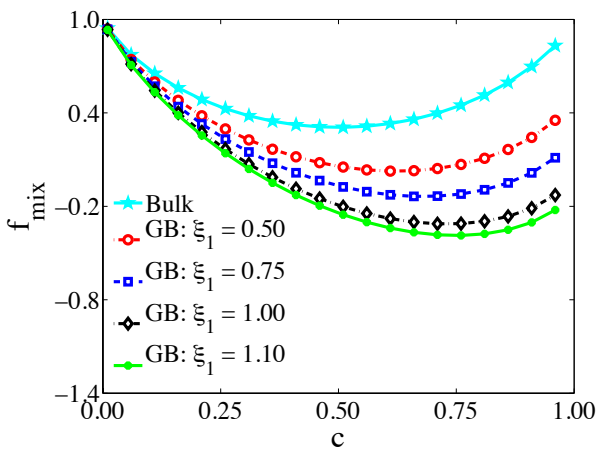

(a)

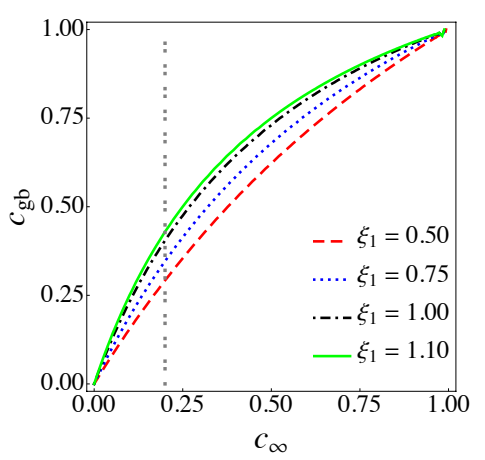

(b)

Figure 4: (Color online). (a) Plots of the chemical component of the free energy, $f_{\text {mix }}$, for the bulk grain, Eq. (4), and GB, Eq. (7), at various values of $\xi_{1}$. Notice that as $\xi_{1}$ increases, the minima of the energy curves shift to larger concentrations. (b) With the aid of Eq. (14), plots of the analytical GB segregation isotherms at various values of $\xi_{1}$, where $c_{g b}=c_{e}\left(\mathbf{r} \mid \phi_{e}(\mathbf{r})=0.5\right)$ is the concentration at the center of the GB. The vertical dashed line indicates the initial concentration employed in the simulations. In both plots, it was assumed that $\xi_{2}=\Omega=0$.

We first start by examining the role of the model parameter $\xi_{1}$, while setting $\xi_{2}=\Omega=0$, on the evolution of the oblong grain and the concentration profile. This corresponds to ideal alloys $(\Omega=0)$, where the GB segregation is uniform $\left(\xi_{1} \neq 0\right)$ with no solute-solute interactions within GBs $\left(\xi_{2}=0\right)$. Initially, the concentration was assumed uniform and constant everywhere $c(\mathbf{r}, t=0)=\langle c\rangle=0.2$. According to Eq. (8), $\xi_{1}$ affects the free energy of mixing within the GB region by changing the value of $G_{B}^{g b}$ relative to the one for bulk grain. Fig. 4(a) shows plots of the chemical component of the free energy, $f_{m i x}$, for the bulk grain and GB regions, Eqs. (4) and (7), respectively, for various values of $\xi_{1}$. It can be seen that increasing $\xi_{1}$ shifts the minima of $f_{\text {mix }}$ towards larger concentration values relative to the bulk grain one. With the aid of Eq. (14), Fig. 4(b) shows plots of the analytical equilibrium GB segregation isotherms at the center of the GB region, where $c_{g b}=c_{e}\left(\mathbf{r} \mid \phi_{e}(\mathbf{r})=0.5\right)$, for various values of $\xi_{1}$. The vertical dashed line indicates the initial uniform concentration employed in the numerical simulations. The GB concentration increases with $\xi_{1}$, nonetheless, large $\xi_{1}$ values are needed to attain GB concentrations that are considerably larger than the far-field one.

Next, we track the temporal evolution of the x-position and concentration 


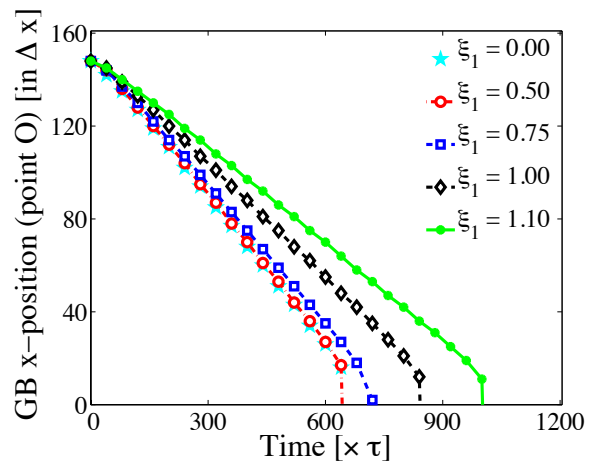

(a)

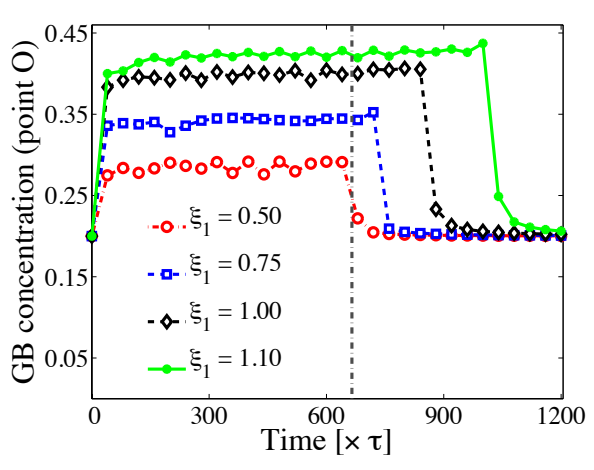

(b)

Figure 5: (Color online). Results for varying $\xi_{1}$ while keeping $\xi_{2}=\Omega=0$. For the GB point $\mathrm{O}$ [cf. Fig. 3(a)], temporal evolution of the (a) x-position relative to the reference frame that is positioned at the center of the oblong grain, and (b) solute concentration field. In panel (b), the sudden drop in the concentration profiles corresponds to complete shrinkage of the oblong grain. The time to complete shrinkage for the system under normal conditions with no GB segregation is indicated by the vertical dashed line.

field of the GB point O [cf. Fig. 3(a)]. Simulation results with various values of $\xi_{1}$ are shown in Fig. 5. The evolution of the x-position relative to the reference frame that is positioned at the center of the oblong grain is depicted in Fig. 5(a), where the case under normal conditions with no GB segregation, i.e., $\xi_{1}=\xi_{2}=0$, is marked by the five-pointed star in cyan. For the $\xi_{1}$ values considered in this study, the spatial evolution is linear in time except at late times, when the oblong grain virtually shrank and both semi-circular ends of the grain were in close proximity to each other. During the evolution, the semi-circular caps maintained a constant local curvature and variations in the driving force were solely due to GB energy and its dependence on segregation parameters. The slopes of the lines in Fig. 5(a) correspond to the velocity of GB point $\mathrm{O}$, which according to Eq. (1), is proportional to the product of GB mobility, energy and curvature; all of which are held constant in the simulations except the GB energy, which varies with $\xi_{1}$. As $\xi_{1}$ increases, the oblong grain shrinks at a slower rate than the system under normal conditions with no GB segregation.

The evolution of the concentration field at point $\mathrm{O}$ is shown in Fig. 5(b). Solute enrichment at the GB increases with $\xi_{1}$, which for the choice of the kinetic parameters in this study, leads to slower dynamics. Simulation results 
for the concentration at GB point $\mathrm{O}$ [cf. Fig. 5(b)] are in agreement with values from the analytical GB segregation isotherms that are calculated using Eq. (14) and depicted in Fig. 4(b). We note that the sudden drop in the concentration profiles [cf. Fig. 5(b)] corresponds to the point in time when the racetrack-shaped grain completely shrank, i.e., there are no GBs present in the system, and the point $\mathrm{O}$ reached the center of the simulation domain. After complete shrinkage of the oblong grain, the concentration profile quickly evolved to reach an equilibrium uniform state, thus leading to the asymptotic behavior of the concentration profiles towards the alloy nominal concentration. The results depicted in Fig. 5 indicate that increased thermodynamic stability is attained at large values of the uniform segregation model parameter, i.e., $\xi_{1}>1.0$. It is worth mentioning here that in the dilute limit with no solute-solute interactions within the GB, i.e., $\xi_{2}=\Omega=0$, our treatment can be mapped onto the one proposed by Li et al. [50] for GB solute drag in binary alloys, where $\xi_{1}$ corresponds to their model parameter $\left(W_{A}-W_{B}\right) / V_{m}$.

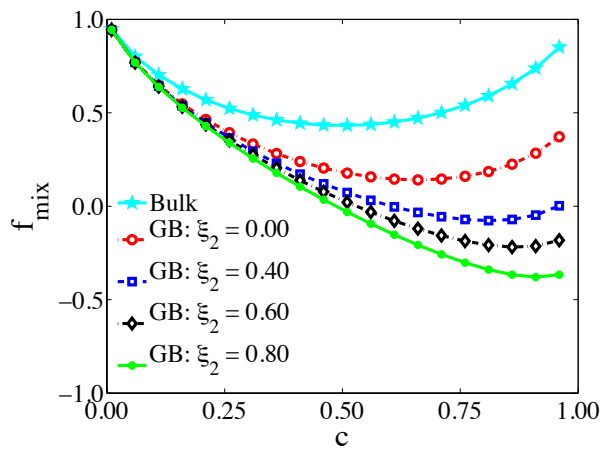

(a)

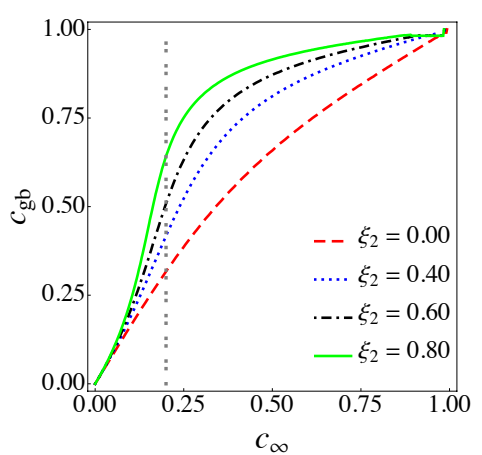

(b)

Figure 6: (Color online). (a) Plots of the chemical component of the free energy, $f_{\text {mix }}$, for the bulk grain, Eq. (4), and GB, Eq. (7), at various values of $\xi_{2}$. Notice that the curvature of the effective GB energy curve decreases as $\xi_{2}$ increases. (b) With the aid of Eq. (14), plots of the analytical GB segregation isotherm at various values of $\xi_{2}$, where $c_{g b}=c_{e}\left(\mathbf{r} \mid \phi_{e}(\mathbf{r})=0.5\right)$ is the concentration at the center of the GB. The vertical dashed line indicates the initial concentration employed in the simulations. In both plots, it was assumed that $\xi_{1}=\Omega=0.5$.

We now turn our attention to solute-solute interactions within the GB, i.e., the model parameter $\xi_{2}$. We examine GB motion and the evolution of 
the concentration field for various values of $\xi_{2}$. Within this treatment, a GB segregation isotherm with non-zero values for both $\xi_{1}$ and $\xi_{2}$ is similar in form to the Fowler-Guggenheim isotherm [25, 29], where $\xi_{2}$ accounts for solute-solute interactions within the GB regions. $\xi_{2}>0$ leads to attractive solute-solute interactions, where the concentration at the GB grows with segregation. On the other hand, repulsive interactions are accounted for via $\xi_{2}<0$, where $\mathrm{GB}$ solute enrichment decreases the propensity to further segregation. A close inspection of Eq. (8) reveals that $\xi_{2}$ controls both the effective GB enthalpic coefficient, $\Omega^{g b}$, and the free energy density of pure $\mathrm{B}, G_{B}^{g b} . \Omega^{g b}$ increases with $\xi_{2}$, which in turn decreases the curvature of the free energy curve. We note that in regular solution treatments, a miscibility gap develops when $\Omega>2.0$, where phase separation leads to the formation of compositional domains and their subsequent growth [15]. Here, we focus on systems, where $f_{m i x}$ is characterized by a single minimum since it is the dependence of GB migration on solute segregation model parameters that we seek to quantify.

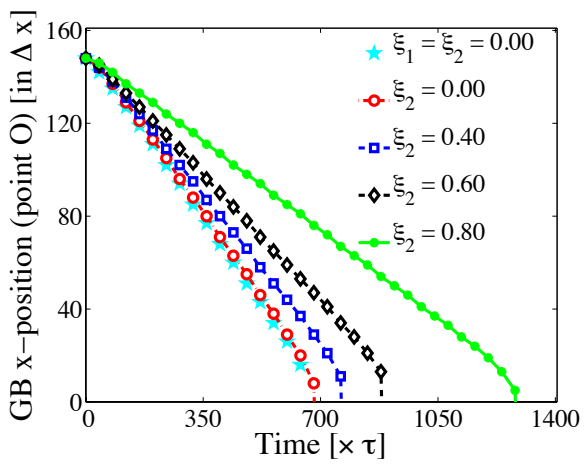

(a)

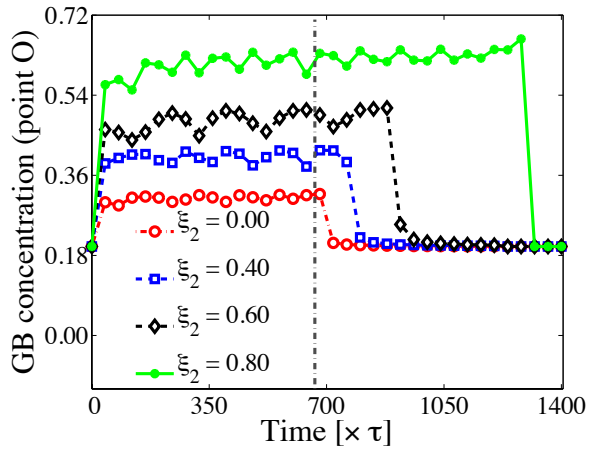

(b)

Figure 7: (Color online). Results for varying $\xi_{2}$ while keeping $\xi_{1}=\Omega=0.5$. For the GB point $\mathrm{O}$ [cf. Fig. 3(a)], temporal evolution of the (a) x-position relative to the reference frame that is positioned at the center of the oblong grain, and (b) solute concentration field. In panel (b), the sudden drop in the concentration profiles corresponds to complete shrinkage of the oblong grain. The time to complete shrinkage for the system under normal conditions with no GB segregation is indicated by the vertical dashed line.

In this study, we set $\left(G_{A}, G_{B}, \Omega, \xi_{1}\right)=(1.0,1.0,0.5,0.5)$ and the initial concentration was assumed uniform with $c(\mathbf{r}, t=0)=\langle c\rangle=0.2$. Plots of the resulting chemical component of the free energy, $f_{m i x}$, for the bulk grain and 
GB for various values of $\xi_{2}$ are shown in Fig. 6(a). It can be seen that the minima of $f_{\text {mix }}$ for the GB shift to larger concentration values as $\xi_{2}$ increases, i.e., stronger attractive interactions. Equilibrium GB segregation isotherms at the center of the GB, where $c_{g b}=c_{e}\left(\mathbf{r} \mid \phi_{e}(\mathbf{r})=0.5\right)$ are shown in Fig. 6(b) for the $\xi_{2}$ values used in this study. The vertical dashed line is drawn at the initial uniform concentration $c(\mathbf{r}, t=0)=\langle c\rangle=0.2$. It is clear that GB enrichment increases nonlinearly with positive $\xi_{2}$. According to Eq. (14), in the limit when $\Omega^{g b}=\Omega+\xi_{2}>2.0$, the GB segregation isotherm becomes multivalued indicating phase transformation within the GB. Several binary alloys were experimentally observed to have values close to this limit [25].

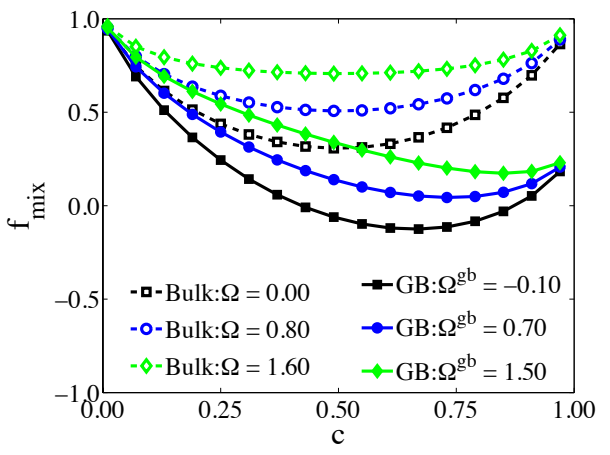

(a)

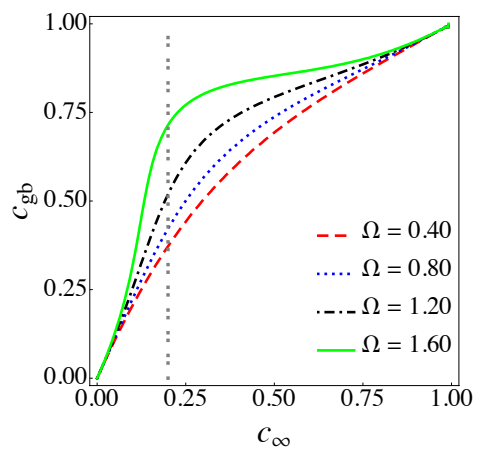

(b)

Figure 8: (Color online). (a) Plots of the chemical component of the free energy, $f_{m i x}$, for the bulk grain, Eq. (4), and GB, Eq. (7), at various values of $\Omega$. By virtue of Eq. (8), the effective GB solute-solute interaction term is written as: $\Omega^{g b}=\Omega+\xi_{2}$. (b) With the aid of Eq. (14), plots of the analytical GB segregation isotherm at various values of $\Omega$, where $c_{g b}=c_{e}\left(\mathbf{r} \mid \phi_{e}(\mathbf{r})=0.5\right)$ is the concentration at the center of the GB. The vertical dashed line indicates the initial concentration employed in the simulation. In both plots, it was assumed that $\left(\xi_{1}, \xi_{2}\right)=(0.8,-0.1)$.

Simulation results for various values of $\xi_{2}$ are shown in Fig. 7, where panel (a) depicts the evolution of the x-position of GB point O [cf. Fig. 3(a)] relative to the reference frame. It evident that increasing $\xi_{2}$, for the parameters $\xi_{1}=\Omega=0.5$ that were chosen here, leads to slower dynamics (improved thermodynamic stability). For example, complete shrinkage of the oblong grain with $\xi_{2}=0.8$ occurs at (simulation) time $\simeq 1300 \tau$ compared to shrinkage time $\simeq 670 \tau$ for the system under normal conditions with no GB segregation, i.e., $\xi_{1}=\xi_{2}=0.0$. The evolution of the concentration field at point $\mathrm{O}$ is in turn shown in Fig. 7(b), where as expected 
increasing $\xi_{2}$ leads to solute enrichment at the GB. A positive value for $\xi_{2}$ corresponds to attractive solute-solute interactions within the GB, where the extent of segregation is enhanced with increasing GB concentration. For $\xi_{2}=(0.0,0.4,0.6,0.8)$, the concentration at point $\mathrm{O}$ attains the asymptotic values $\sim(0.31,0.4,0.47,0.62)$, respectively, which are in agreement with the values from the analytical GB segregation isotherm predicted by Eq. (14) and shown in Fig. 6(b). The sudden drop in the concentration profiles at late times [cf. Fig. $7(\mathrm{~b})]$ is due to the fact that when the oblong grain completely shrinks, i.e., no GBs present in the system, the point $\mathrm{O}$ reaches the center of the simulation box. Then, the concentration field quickly evolves to the equilibrium uniform state. It is worth noting that a slight fluctuation in the reported concentration values at point $\mathrm{O}$ can be observed in Fig. 7(b). This is primarily due to the discreteness of the numerical solution. The GB center point (point $\mathrm{O}$ ) may not coincide with an actual pixel and in such cases, the concentration value at the closest numerical grid point was recorded. The observed fluctuation in the concentration values around the analytical ones was roughly on the order of 0.04 .

Finally, we consider non-ideal alloys by varying the heat of mixing parameter, $\Omega$. Here, we let $\left(G_{A}, G_{B}, \xi_{1}, \xi_{2}\right)=(1.0,1.0,0.8,-0.1)$ and set $\Omega=$ $(0.0,0.4,0.8,1.2,1.6)$, which by virtue of Eq. (8) yields an effective GB enthalpic coefficient $\Omega^{g b}=(-0.1,0.3,0.7,1.1,1.5)$, respectively. As an illustration, plots of the resulting chemical component of the free energy, $f_{\text {mix }}$, curves for both bulk and GB regions with $\Omega=(0.0,0.8,1.6)$ are shown in Fig. 8(a). According to Eq. (8), $G_{B}^{g b}$ dictates the shift in the effective GB free energy curves and is set through $\xi_{1}$ and $\xi_{2}$. On the other hand, increasing $\Omega$ and subsequently $\Omega^{g b}$ decreases the curvature of the free energy curves. Fig. 8(b) shows the analytical GB segregation isotherms at various values of $\Omega$, where the GB concentration, $c_{g b}$, is calculated at the center of the GB, i.e., $\phi_{e}=0.5$. It can be seen that the increase in GB concentration, $c_{g b}$, with $\Omega$ is more pronounced at large far-field bulk concentrations, $c_{\infty}$. We also note that the shape of the analytical GB segregation isotherm at large $\Omega$ [cf. Fig. 8(b)] is similar to the one predicted, via thermodynamic treatments, for $\mathrm{Ni}-\mathrm{P}$ and Ni-W alloys [32].

Simulation results for the evolution of the oval racetrack-shaped grain [cf. Fig. 3(a)] for various values of $\Omega$ are shown in Fig. 9, where panel (a) is a plot of the temporal evolution of the x-position of GB point $\mathrm{O}$. It is evident that 


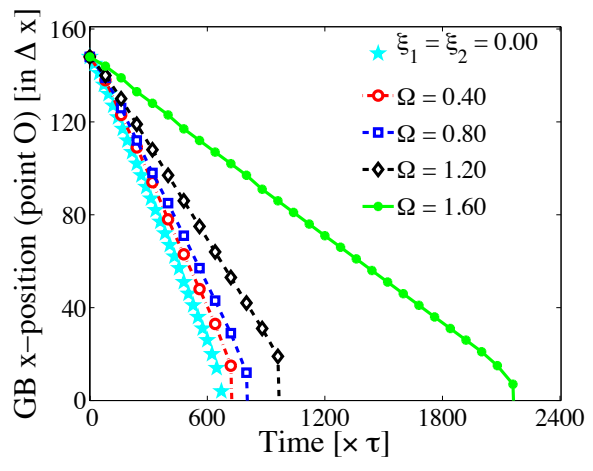

(a)

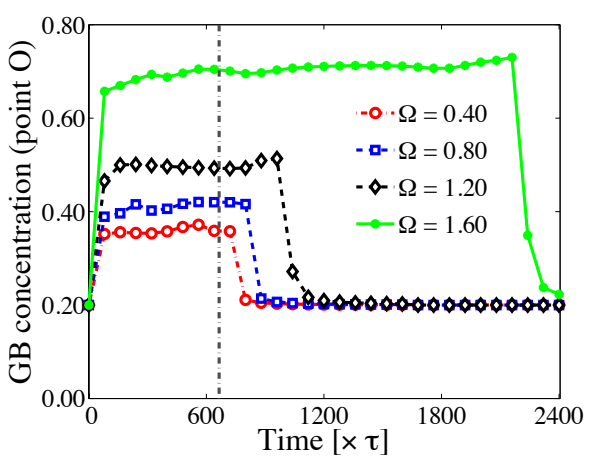

(b)

Figure 9: (Color online). Results for varying the heat of mixing parameter, $\Omega$, while keeping $\left(\xi_{1}, \xi_{2}\right)=(0.8,-0.1)$. For the GB point $\mathrm{O}$ [cf. Fig. 3(a)], temporal evolution of the (a) x-position relative to the reference frame that is positioned at the center of the oblong grain, and (b) solute concentration field. In panel (b), the sudden drop in the concentration profiles corresponds to complete shrinkage of the oblong grain. The time to complete shrinkage for the system under normal conditions with no GB segregation is indicated by the vertical dashed line.

increasing $\Omega$ leads to slower dynamics. For example, the time to complete shrinkage of the oblong grain is $2200 \tau$ for the system with $\Omega=1.6$, compared to $670 \tau$ for the one under normal conditions with no GB segregation, i.e., $\xi_{1}=\xi_{2}=0$. Moreover, the evolution of the GB point $\mathrm{O}$ depends nonlinearly on $\Omega$. This can be explained by examining Eqs. (16) and (17), where it can be seen that the GB energy, $\gamma_{g b}$, is non-linearly dependent on the thermodynamic model parameters including $\Omega$.

Next, Fig. 9(b) depicts the evolution of the concentration profile at the GB point $\mathrm{O}$ for various values of $\Omega$. Solute enrichment at the GB increases with $\Omega$, which in turn leads to slower shrinkage dynamics. The reason for this can be understood as follows. Within the treatment of regular solutions, $\Omega>0$ indicates that the A-B bond energy is larger than the average of A-A and B-B bond energies and increasing $\Omega$ leads to unfavorable mixing states [15]. Therefore, solute atoms segregate to GBs due to both the segregation energy that is set by $\xi_{1}$ and $\xi_{2}$ and the energetically unfavorable mixing state within the bulk due to large and positive $\Omega$ values. Similar to the cases shown in Figs. 5(b) and 7(b), the sudden drop in the concentration profiles observed in Fig. 9(b) is due to the fact that when the oblong grain 
completely shrinks, $\phi$ becomes constant everywhere and the point $\mathrm{O}$ reaches the center of the simulation box. The concentration profile then quickly evolves to the equilibrium uniform state. The results depicted in Fig. 9 indicate that, for the model parameters chosen here, improved thermodynamics stability occurs in systems with relatively large heat of mixing.

\subsection{Polycrystalline systems}

In this section, we turn our attention to polycrystalline systems and demonstrate the capability of the model to examine and quantitatively describe GB solute segregation. In contrast to the controlled two-grain geometry depicted in Fig. 3(a), polycrystalline aggregates are characterized by a distribution of grain sizes, i.e., effective radii for the grains, which according to Eq. (1) leads to a wide range for the driving force for grain growth. Therefore, in this study we quantify such systems by constructing grain size distributions and extracting from them geometric metrics such as average grain size, $\left\langle D_{g}\right\rangle$.

An initial configuration of a representative system was prepared by solving the evolutionary equations for the grain OPs, Eqs. (10), with no GB solute segregation, i.e., $\xi_{1}=\xi_{2}=0$, for a few time steps until the desired microstructure was reached. In this preparation step, the initial values for the OPs, $\phi_{i}(\mathbf{r}, t=0)=0.5+\eta(\mathbf{r})$, where $\eta \in(-0.5,0.5)$ is a uniform random number. Systems were generated with $N_{x}=N_{y}=1024, \Delta t=0.0001$ and $n_{\phi}=50$. Other parameters used in the simulations presented in this section were: $\left(W_{\phi}, \epsilon, L, M\right)=(0.4,1.9,1.0,1.0)$. For better visualization of the grain morphology and GB regions, we define the scalar field $\Xi(\mathbf{r}, t)$, such that

$$
\Xi(\mathbf{r}, t)=\sum_{i=1}^{n_{\phi}} \phi_{i}^{2}(\mathbf{r}, t)\left[1-\phi_{i}(\mathbf{r}, t)\right]^{2},
$$

where in the grain interior far from GBs, $\Xi=0$. Within a GB region, where typically two order parameters overlap $\left\{\phi_{i}=\phi_{j}=0.5, \phi_{k \neq i, j}=0, k=\right.$ $\left.1, \cdots, n_{\phi}\right\}, \Xi \simeq 1 / 8$. The initial microstructure used in the GB segregation study presented here is shown in Fig. 10(a), where the number of grains was 573. Motivated by the results presented in Section 4.1, we employ GB segregation model parameters with large $\Omega$. Namely, we set $\left(\xi_{1}, \xi_{2}, \Omega\right)=(0.8,-0.1,1.6)$, where the resulting 
1D analytical GB segregation isotherm is depicted by the solid green line in Fig. 8(b). Furthermore, we consider two polycrystalline systems having the same initial microstructure [cf. Fig. 10(a)] and GB segregation parameters but differing in the initial solute concentration $c(\mathbf{r}, t=0)=\langle c\rangle$. Here, we examined systems with $\langle c\rangle=0.2$ and 0.35 , and the results were compared to the case under normal grain growth conditions with no GB segregation, i.e., $\xi_{1}=\xi_{2}=0$.

First, we qualitatively examine the microstructural evolution of polycrystalline systems. Panels (b) through (d) of Fig. 10 depict the resulting microstructures at (simulation) time $=1200 \tau$ for the cases with no GB segregation [panel (b)], and the ones with segregation parameters $\left(\xi_{1}, \xi_{2}, \Omega\right)=(0.8,-0.1,1.6)$ with $\langle c\rangle=0.2$ [panel (c)] and 0.35 [panel (d)]. First, by comparing Figs. 10(c) and (d) with (b) it can be seen that GB segregation yields microstructures with grains that are finer than the one under normal growth conditions. Moreover, for the same GB segregation parameters, varying the initial concentration in the alloy results in morphologies that differ in both shape and size [cf. Figs. 10(c) and (d)]. In alloys that favor strong GB segregation, an increase in the initial alloy concentration results in large accumulation of solute at the GBs, thereby widening GB regions. This trend can also be seen in our 1D analytical treatment, where Figs. 2(b) and (d) depict the dependence of GB width on far-field solute concentrations. Indeed, recent Monte Carlo alloy simulations of equilibrium configurations in nanostructures [37] reveal morphologies with wide GB regions similar to the one depicted in Fig. 10(d). To examine the shapes of the concentration field and grain OPs across GB regions, their spatial profiles were extracted along the red A-B line, which is shown in Fig. 10(d), and the results are depicted in Fig. 10(e). It can be seen that the concentration peaks at the center of the GBs and changes smoothly across the wide GB regions to the equilibrium value in the bulk grains. Additionally, the grain OPs maintained their diffusive profiles across GB regions.

To quantify the results shown in Fig. 10, the $\Xi$ field was mapped onto an Ising model. Within this binary description, GB regions, which are characterized by diffuse grain OPs, were assigned a numerical label that is different than the one given for bulk grains with constant grain OPs. Next, the Hoshen-Kopelman cluster la- 


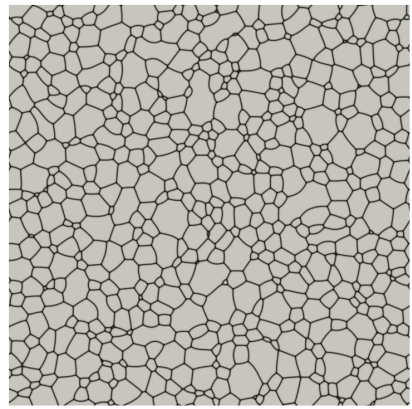

(a)

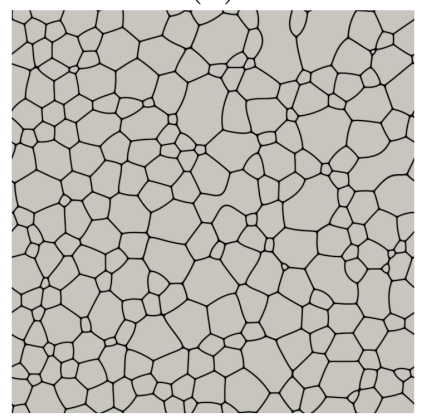

(c)

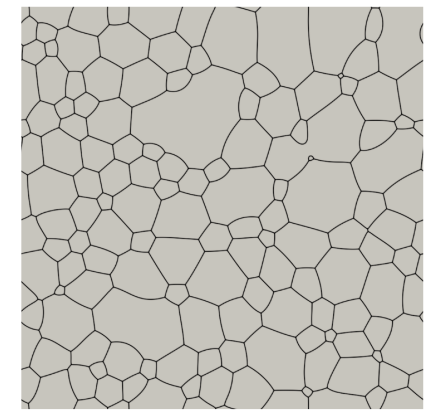

(b)

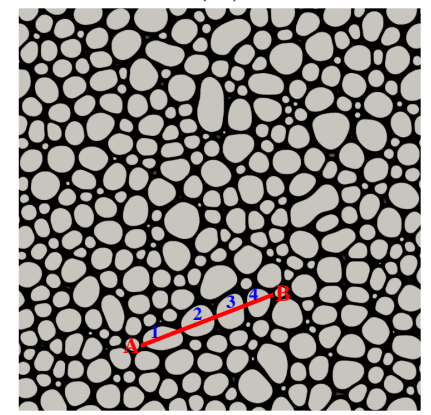

(d)

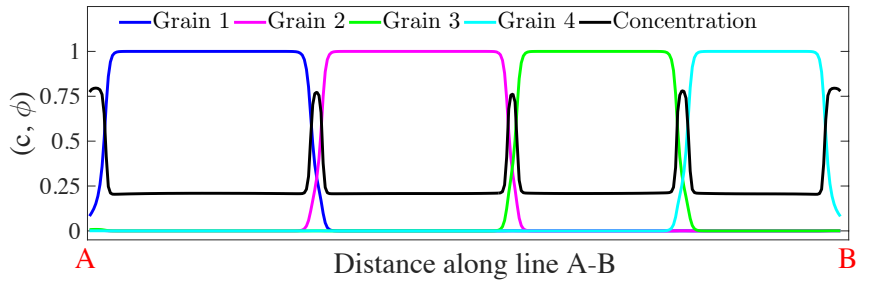

(e)

Figure 10: (Color online). (a) The initial $(t=0)$ microstructure used in the GB segregation study of polycrystalline systems. Snapshots of the grain microstructures at (simulation) time $=1200 \tau$ for the system with (b) no GB solute segregation, i.e., $\xi_{1}=\xi_{2}=0$, and the ones with $\left(\xi_{1}, \xi_{2}, \Omega\right)=(0.8,-0.1,1.6)$ with an initial solute concentration $c(\mathbf{r}, t=0)=\langle c\rangle$ of (c) 0.2 and (d) 0.35 . (e) At time $=1200 \tau$, spatial profile of the concentration field and grain OPs along the red A-B line that is drawn across four grains in panel (d). The grains are numerically labeled for easy identification. In panels [(a)-(d)], grey (black) denote bulk grain (GB regions).

beling algorithm [66] was modified to incorporate periodic bound- 


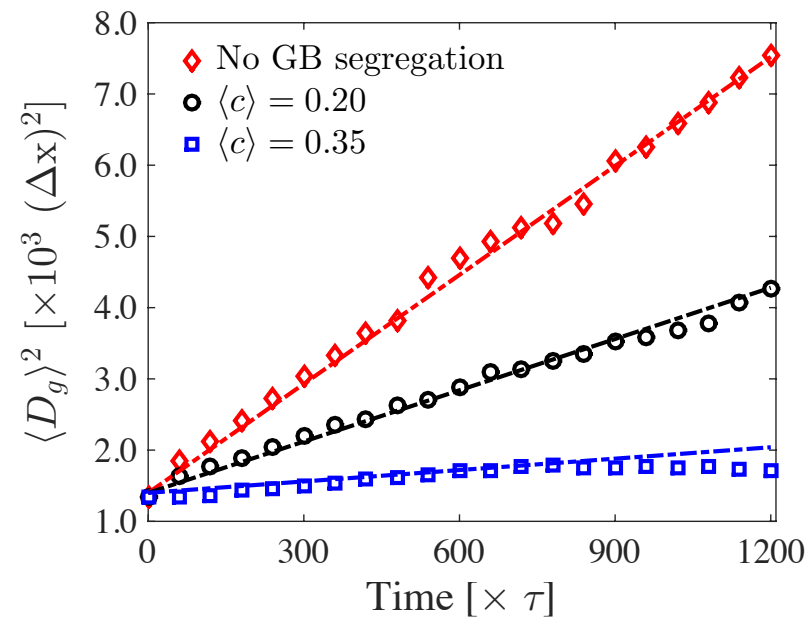

Figure 11: (Color online). The temporal evolution of $\left\langle D_{g}\right\rangle^{2}$, where $\left\langle D_{g}\right\rangle$ denotes the average grain size, for binary alloys with GB segregation model parameters $\left(\xi_{1}, \xi_{2}, \Omega\right)=$ $(0.8,-0.1,1.6)$ and an initial solute concentration $c(\mathbf{r}, t=0)=\langle c\rangle=0.2$ and 0.35 . The case under normal grain growth with no GB segregation is shown for comparison. The straight dash-dot lines are drawn to guide the eye.

ary conditions and then used to construct the grain size distribution and the corresponding average grain size, $\left\langle D_{g}\right\rangle$, i.e., the first moment of the grain size distribution, as these microstructures evolved over time. As is typically done in grain growth studies [67], the temporal evolution of $\left\langle D_{g}\right\rangle^{2}$, which is a measure of average grain area, $\left\langle A_{g}\right\rangle$, where $\left\langle A_{g}\right\rangle \propto\left\langle D_{g}\right\rangle^{2}$, is shown in Fig. 11. At any given time, alloy systems with GB solute segregation are characterized by an average grain area, $\left\langle D_{g}\right\rangle^{2}$, that is smaller than the one with no GB segregation. Furthermore, for the GB segregation parameters chosen in this study, increasing the initial solute concentration leads to a reduction in grain growth dynamics. For example, at a simulation time of $1200 \tau$, the alloy with $\langle c\rangle=0.35$ is characterized by an average grain area, $\left\langle D_{g}\right\rangle^{2}$, that is roughly three times smaller than the one with $\langle c\rangle=0.2$. The trends observed is Fig. 11 suggest that GB segregation can serve as a stabilizing mechanism against homogenization processes, where the proper choice of the alloying element and solute content in these systems dictates the rate of 


\section{grain growth relative to pure systems with no GB segregation.}

\section{Summary and Future Outlook}

GB segregation in binary alloys has been proposed as a stabilization mechanism against grain growth and homogenization processes. However, the thermodynamic and kinetic aspects of this mechanism have not been fully explored and quantified. In this work, we developed a phase field model for GB solute segregation in polycrystalline binary alloys with the main aim of providing a quantitative description of the thermodynamic aspects of GB segregation. More specifically, we explored regimes far from ideal scenarios, where solute-solute interactions within GBs are non-trivial and the reduction in GB energy due to segregation can be large.

Within this mesoscale treatment, the solute concentration field was coupled to a set of structural order parameters that describe grains with different crystallographic orientations. The model provides a general framework that is capable of accounting for the dependence of GB energy on solute segregation and capturing solute-solute interactions within GB regions. A one-dimensional analytical treatment of the model was presented, where the resulting GB segregation isotherm was mapped onto a form similar to the Fowler-Guggenheim isotherm. In ideal and dilute systems, the resulting isotherm reduces to the classic McLean one, which is used in current mesoscale treatments to examine dynamic solute drag. The dependencies of the GB energy and width on the segregation model parameters were explored, where regimes of considerable reduction in GB energy were revealed. A parametric study on two-dimensional systems was conducted in order to survey the phase space of GB segregation model parameters and examine grain growth dynamics. Simulation results revealed regimes, where increased thermodynamic stability was attained. More specifically, it was found that stability is highly influenced by the degree of non-ideality and the total solute content in these systems.

Finally, we are currently examining the role of anisotropy in GB energy, mobility and segregation tendency on grain growth dynamics. This of course requires detailed analysis, via atomistic simulations for example, in order to correctly quantify the dependence of these anisotropies on GB type and character. Moreover, we are utilizing the present model to investigate GB segregation in systems, where phase separation occurs in conjunction with grain growth, and we hope to report on these results in a future publication. 


\section{Acknowledgments}

The authors would like to thank Dr. Blythe G. Clark for useful discussions. This work was funded by the U.S. Department of Energy, Office of Basic Energy Sciences, Division of Materials Sciences and Engineering. Sandia National Laboratories is a multi-program laboratory managed and operated by Sandia Corporation, a wholly owned subsidiary of Lockheed Martin Corporation, for the U.S. Department of Energys National Nuclear Security Administration under contract DE-AC04-94AL85000.

\section{Appendix A. Equilibrium Phase Field Profile}

Here, we derive the equilibrium profile for the phase field, $\phi_{e}$. First, we start with the governing equation for the stationary phase field, i.e., Eq. (12), which is written as

$$
W_{\phi} \frac{d f_{\text {grain }}}{d \phi_{e}}-\epsilon^{2} \nabla^{2} \phi_{e}-\left(\xi_{1} c_{e}+\xi_{2} c_{e}^{2}\right) \frac{d f_{\text {grain }}}{d \phi_{e}}=0,
$$

where $f_{\text {grain }}\left(\phi_{e}\right)=16 \phi_{e}^{2}\left(1-\phi_{e}\right)^{2}$. Multiplying both sides of Eq. (A.1) with $d \phi_{e} / d x$ and integrating yields

$$
\frac{\epsilon^{2}}{2}\left(\frac{d \phi_{e}}{d x}\right)^{2}=W_{\phi} f_{\text {grain }}\left(\phi_{e}\right)-\int_{0}^{\phi}\left(\xi_{1} c_{e}+\xi_{2} c_{e}^{2}\right) \frac{d f_{\text {grain }}}{d \phi_{e}} d \phi_{e}
$$

where again $c_{e}=c_{e}(x)$. Integration by parts the last term in Eq. (A.2) leads to

$$
\begin{aligned}
\int_{0}^{\phi}\left(\xi_{1} c_{e}+\xi_{2} c_{e}^{2}\right) \frac{d f_{\text {grain }}}{d \phi_{e}} d \phi_{e} & =\left(\xi_{1} c_{e}+\xi_{2} c_{e}^{2}\right) f_{\text {grain }}\left(\phi_{e}\right) \\
& -\int_{c_{\infty}}^{c}\left(\xi_{1}+2 \xi_{2} c_{e}\right) f_{\text {grain }}\left(\phi_{e}\right) d c_{e} .
\end{aligned}
$$

The integral on the right hand side of Eq. (A.3) can be simplified with the aid of Eq. (13) to give

$$
\begin{aligned}
\int_{c_{\infty}}^{c}\left(\xi_{1}+2 \xi_{2} c_{e}\right) f_{\text {grain }}\left(\phi_{e}\right) d c_{e} & =\int_{c_{\infty}}^{c}\left[\left.\frac{\partial f_{\text {mix }}}{\partial c}\right|_{c_{e}}-\left.\frac{\partial f_{\text {mix }}}{\partial c}\right|_{c_{\infty}}\right] d c_{e} \\
& =f_{\text {mix }}(c)-f_{\text {mix }}\left(c_{\infty}\right)-\left.\frac{\partial f_{m i x}}{\partial c}\right|_{c_{\infty}}\left(c-c_{\infty}\right) .
\end{aligned}
$$


Next, we combine Eqs. (A.2)-(A.4) to give the following for the equilibrium profile of the phase field

$$
\begin{aligned}
\frac{\epsilon^{2}}{2}\left(\frac{d \phi_{e}}{d x}\right)^{2} & =W_{\phi} f_{\text {grain }}\left(\phi_{e}\right)-\left(\xi_{1} c_{e}+\xi_{2} c_{e}^{2}\right) f_{\text {grain }}\left(\phi_{e}\right) \\
& +f_{\text {mix }}(c)-f_{\text {mix }}\left(c_{\infty}\right)-\left.\frac{\partial f_{\text {mix }}}{\partial c}\right|_{c_{\infty}}\left(c-c_{\infty}\right) .
\end{aligned}
$$

Finally, the last three terms on the right hand side of Eq. (A.5) are evaluated with the aid of Eq. (4) for the free energy of the alloy to yield the following equation for the equilibrium phase field, $\phi_{e}$

$$
\begin{aligned}
\frac{\epsilon^{2}}{2}\left(\frac{d \phi_{e}}{d x}\right)^{2}= & W_{\phi} f_{\text {grain }}\left(\phi_{e}\right)-\left(\xi_{1} c_{e}+\xi_{2} c_{e}^{2}\right) f_{\text {grain }}\left(\phi_{e}\right)+ \\
& \frac{R T}{V_{m}}\left[c_{e} \ln \left(\frac{c_{e}}{c_{\infty}}\right)+\left(1-c_{e}\right) \ln \left(\frac{1-c_{e}}{1-c_{\infty}}\right)\right]-\Omega\left(c_{e}-c_{\infty}\right)^{2} .
\end{aligned}
$$

\section{Appendix B. Grain Boundary Properties}

Based on the governing equation for the equilibrium phase field profile, $\phi_{e}$, i.e., Eq. (A.6), we examine the dependencies of the GB energy and width on the segregation model parameters $\xi_{1}$ and $\xi_{2}$. GB energy is defined as [27, $54,56]$

$$
\gamma_{g b}=\Delta \mathcal{F}_{t o t}-\left.\Gamma \frac{\partial f_{\text {mix }}}{\partial c}\right|_{c_{\infty}},
$$

where the first term on the right hand side denotes the difference in free energy between a system with a GB and one without at the equilibrium concentration. The second term on the right hand side assumes a Gibbsian adsorption isotherm with $\Gamma=\int_{-\infty}^{+\infty} d x\left(c_{e}-c_{\infty}\right)$ being the solute excess at the GB. Here, it is implicitly assumed that the molar volumes of the host and alloying elements are the same. With the aid of Eq. (3), the GB energy is written as

$$
\begin{aligned}
\gamma_{g b} & =\int_{-\infty}^{+\infty} d x\left[f_{\text {mix }}\left(c_{e}\right)+W_{\phi} f_{\text {grain }}\left(\phi_{e}\right)+\frac{\epsilon^{2}}{2}\left(\frac{d \phi_{e}}{d x}\right)^{2}\right. \\
& \left.-\left(\xi_{1} c_{e}+\xi_{2} c_{e}^{2}\right) f_{\text {grain }}\left(\phi_{e}\right)\right]-\int_{-\infty}^{+\infty} d x f_{\text {mix }}\left(c_{\infty}\right) \\
& -\left.\frac{\partial f_{\text {mix }}}{\partial c}\right|_{c_{\infty}} \int_{-\infty}^{+\infty} d x\left(c_{e}-c_{\infty}\right) .
\end{aligned}
$$


By utilizing Eq. (A.5), we realize that

$$
\begin{array}{r}
f_{\text {mix }}\left(c_{e}\right)-f_{\text {mix }}\left(c_{\infty}\right)-\left.\frac{\partial f_{\text {mix }}}{\partial c}\right|_{c_{\infty}}\left(c_{e}-c_{\infty}\right)= \\
\frac{\epsilon^{2}}{2}\left(\frac{d \phi_{e}}{d x}\right)^{2}-W_{\phi} f_{\text {grain }}\left(\phi_{e}\right)+\left(\xi_{1} c_{e}+\xi_{2} c_{e}^{2}\right) f_{\text {grain }}\left(\phi_{e}\right),
\end{array}
$$

which, when combined with Eq. (B.2), yields the following for the GB energy

$$
\gamma_{g b}=\epsilon^{2} \int_{-\infty}^{+\infty}\left(\frac{d \phi_{e}}{d x}\right)^{2} d x=\epsilon^{2} \int_{0}^{1} \frac{d \phi_{e}}{d x} d \phi_{e}
$$

where $d \phi_{e} / d x$ is given by Eq. (A.6).

Next, the GB width $\delta_{g b}$ is defined as the distance by which the phase field, $\phi_{e}$, changes from $\phi_{i n}$ to $\phi_{\text {out }}$, where $\phi_{\text {in }}$ and $\phi_{\text {out }}$ are lower and upper bounds on the phase field, $\phi_{e}$. A suitable choice for $\left(\phi_{\text {in }}, \phi_{\text {out }}\right)$ is $(0.1,0.9)[50]$ and the GB width is calculated according to $[54]$

$$
\delta_{g b} \simeq \int_{\phi_{i n}}^{\phi_{o u t}} \frac{d x}{d \phi_{e}} d \phi_{e}
$$

\section{References}

[1] H. Gleiter, Nanocrystalline materials, Prog. Mater. Sci. 33 (1989) 223315.

[2] M. Meyers, A. Mishra, D. Benson, Mechanical properties of nanocrystalline materials, Prog. Mater. Sci. 51 (2006) 427-556.

[3] L. Lu, Y. Shen, X. Chen, L. Qian, K. Lu, Ultrahigh strength and high electrical conductivity in copper, Science 304 (2004) 422-426.

[4] R. Birringer, Nanocrystalline materials, Mat Sci. Eng. A 117 (1989) 33-43.

[5] R. Ferrando, J. Jellinek, R. Johnston, Nanoalloys: from theory to applications of alloy clusters and nanoparticles., Chem. Rev. 108 (2008) 845-910. 
[6] J. Driver, Stability of nanostructured metals and alloys, Scripta Mater. 51 (2004) 819-823.

[7] R. Andrievski, Review of thermal stability of nanomaterials, J. Mater. Sci. 49 (2013) 1449-1460.

[8] R. H. Castro, On the thermodynamic stability of nanocrystalline ceramics, Mater. Lett. 96 (2013) 45-56.

[9] K. Darling, M. Tschopp, B. VanLeeuwen, M. Atwater, Z. Liu, Mitigating grain growth in binary nanocrystalline alloys through solute selection based on thermodynamic stability maps, Comp. Mater. Sci. 84 (2014) $255-266$.

[10] M. Atwater, R. Scattergood, C. Koch, The stabilization of nanocrystalline copper by zirconium, Mat Sci. Eng. A 559 (2013) 250-256.

[11] J. Weertman, Retaining the nano in nanocrystalline alloys, Science 337 (2012) 921-922.

[12] M. Ames, J. Markmann, R. Karos, A. Michels, A. Tschöpe, R. Birringer, Unraveling the nature of room temperature grain growth in nanocrystalline materials, Acta Mater. 56 (2008) 4255-4266.

[13] L. Lu, N. Tao, L. Wang, B. Ding, K. Lu, Grain growth and strain release in nanocrystalline copper, J. App. Phys. 89 (2001) 6408-6414.

[14] R. W. Ballufi, S. M. Allen, W. C. Carter, Kinetics of Materials, Wiley, 2005.

[15] D. A. Porter, K. E. Easterling, Phase transformations in metals and alloys, 2nd ed., CRC Press, 2004.

[16] C. Koch, Structural nanocrystalline materials: an overview, J. Mater. Sci. 42 (2007) 1403-1414.

[17] J. Trelewicz, C. Schuh, Grain boundary segregation and thermodynamically stable binary nanocrystalline alloys, Phys. Rev. B 79 (2009) 094112. 
[18] K. Lucke, K. Detert, A quantitative theory of grain-boundary motion and recrystallization in metals in the presence of impurities, Acta Metall. 5 (1957) 628-637.

[19] J. W. Cahn, The impurity-drag effect in grain boundary motion, Acta Metall. 10 (1962) 789-798.

[20] M. Hillert, Solute drag, solute trapping and diffusional dissipation of gibbs energy, Acta Mater. 47 (1999) 4481-4505.

[21] C. Smith, Grains, phases and interfaces - an interpretation of microstructure, Trans Am. Inst. Min. Engrs. 175 (1948) 15-51.

[22] M. Hillert, Inhibition of grain-growth by 2nd-phase particles, Acta Metall. 36 (1988) 3177-3181.

[23] M. Rosen, J. Kunjappu, Surfactants and Interfacial Phenomena, 4th ed., Wiley, 2012.

[24] S. Lin, Diffusion-controlled surfactant adsorption studied by pendant drop digitization, AIChE Journal 36 (1990) 1785-1795.

[25] P. Lejček, Grain Boundary Segregation in Metals, Springer, 2010.

[26] D. McLean, Grain Boundaries in Metals, Clarendon Press, 1957.

[27] A. P. Sutton, R. W. Balluffi, Interfaces in Crystalline Materials, Clarendon Press, 1996.

[28] M. Guttmann, D. McLean, Grain boundary segregation in multicomponent systems, in: W. C. Johnson, J. M. Blakely (Eds.), Interfacial Segregation, ASM, Metals Park, 1979, pp. 261-348.

[29] R. Fowler, E. Guggenheim, Statistical Thermodynamics, Cambridge University Press, 1939.

[30] J. Weissmüller, Alloy effects in nanostructures, Nanostructured Mater. 3 (1993) 261-272.

[31] A. Detor, C. Schuh, Tailoring and patterning the grain size of nanocrystalline alloys, Acta Mater. 55 (2007) 371-379. 
[32] A. Detor, C. Schuh, Grain boundary segregation, chemical ordering and stability of nanocrystalline alloys: Atomistic computer simulations in the Ni-W system, Acta Mater. 55 (2007) 4221-4232.

[33] A. Detor, C. Schuh, Microstructural evolution during the heat treatment of nanocrystalline alloys, J. Mater. Res. 22 (2011) 3233-3248.

[34] K. Darling, A. Roberts, Y. Mishin, S. Mathaudhu, L. Kecskes, Grain size stabilization of nanocrystalline copper at high temperatures by alloying with tantalum, J. Alloys and Comp. 573 (2013) 142-150.

[35] K. Darling, B. VanLeeuwen, J. Semones, C. Koch, R. Scattergood, L. Kecskes, S. Mathaudhu, Stabilized nanocrystalline iron-based alloys: Guiding efforts in alloy selection, Mat Sci. Eng. A 528 (2011) 4365-4371.

[36] J. Dake, C. Krill III, Sudden loss of thermal stability in fe-based nanocrystalline alloys, Scripta Mater. 66 (2012) 390-393.

[37] T. Chookajorn, C. Schuh, Nanoscale segregation behavior and hightemperature stability of nanocrystalline $\mathrm{W}-20$ at.\% Ti, Acta Mater. 73 (2014) 128-138.

[38] C. Krill III, H. Ehrhardt, R. Birringer, Thermodynamic stabilization of nanocrystallinity, Z. Metallkd 96 (2005) 1134-1141.

[39] T. Chookajorn, H. Murdoch, C. Schuh, Design of stable nanocrystalline alloys., Science 337 (2012) 951-953.

[40] H. Murdoch, C. Schuh, Stability of binary nanocrystalline alloys against grain growth and phase separation, Acta Mater. 61 (2013) 2121-2132.

[41] H. Murdoch, C. Schuh, Estimation of grain boundary segregation enthalpy and its role in stable nanocrystalline alloy design, J. Mater. Res. 28 (2013) 2154-2163.

[42] M. Saber, H. Kotan, C. Koch, R. Scattergood, Thermodynamic stabilization of nanocrystalline binary alloys, J. App. Phys. 113 (2013) 063515 .

[43] P. Millett, R. Selvam, A. Saxena, Molecular dynamics simulations of grain size stabilization in nanocrystalline materials by addition of dopants, Acta Mater. 54 (2006) 297-303. 
[44] A. Seki, D. Seidman, Y. Oh, S. M. Foiles, Monte-Carlo simulations of segregation at [001] twist boundaries in a $\mathrm{Pt}(\mathrm{Au})$ alloy. 1. results, Acta Metall. Mater. 39 (1991) 3167-3177.

[45] M. Hashimoto, Y. Ishida, R. Yamamoto, M. Doyama, Atomistic studies of grain-boundary segregation in Fe-P and Fe-B alloys. 1. atomicstructure and stress-distribution, Acta Metall. 32 (1984) 1-11.

[46] X. Liu, W. Xu, S. M. Foiles, J. Adams, Atomistic studies of segregation and diffusion in Al-Cu grain boundaries, App. Phys. Lett. 72 (1998) $1578-1580$.

[47] P.-R. Cha, S. Kim, D.-H. Yeon, J.-K. Yoon, A phase field model for the solute drag on moving grain boundaries, Acta Mater. 50 (2002) $3817-3829$.

[48] K. Grönhagen, J. Ågren, Grain-boundary segregation and dynamic solute drag theory - a phase-field approach, Acta Mater. 55 (2007) 955960 .

[49] S. Kim, Y. Park, Grain boundary segregation, solute drag and abnormal grain growth, Acta Mater. 56 (2008) 3739-3753.

[50] J. Li, J. Wang, G. Yang, Phase field modeling of grain boundary migration with solute drag, Acta Mater. 57 (2009) 2108-2120.

[51] T. Heo, S. Bhattacharyya, L.-Q. Chen, A phase field study of strain energy effects on solutegrain boundary interactions, Acta Mater. 59 (2011) 7800-7815.

[52] F. Abdeljawad, B. Voelker, R. Davis, R. M. McMeeking, M. Haataja, Connecting microstructural coarsening processes to electrochemical performance in solid oxide fuel cells: An integrated modeling approach, J. Power Sources 250 (2014) 319-331.

[53] Y. Wang, Computer modeling and simulation of solid-state sintering: A phase field approach, Acta Mater. 54 (2006) 953-961.

[54] J. W. Cahn, J. E. Hilliard, Free energy of a nonuniform system. i. interfacial free energy, J. Chem. Phys. 28 (1958) 258-267. 
[55] S. M. Allen, J. W. Cahn, A microscopic theory for antiphase boundary motion and its application to antiphase domain coarsening, Acta Metall. 27 (1979) 1085-1095.

[56] A. Wheeler, W. Boettinger, G. McFadden, Phase-field model of solute trapping during solidification, Phys. Rev. E 47 (1993) 1893-1909.

[57] Wolfram, Mathematica v9.0, 2014. URL: http://www. wolfram. com/mathematica/new-in-9/.

[58] D. Prokoshkina, V. Esin, G. Wilde, S. Divinski, Grain boundary width, energy and self-diffusion in nickel: Effect of material purity, Acta Materialia 61 (2013) 5188-5197.

[59] F. Abdeljawad, Mesoscale modeling of heterogeneous materials systems: from solid oxide fuel cells to bulk metallic glasses, Ph.D. thesis, Princeton University, 2013.

[60] J. Deng, Second order accuracy in phase field modeling of normal grain growth, Met. Mater. Int. 18 (2012) 745-749.

[61] M. Upmanyu, R. Smith, D. Srolovitz, Atomistic simulation of curvature driven grain boundary migration, Inter. Sci. 6 (1998) 41-58.

[62] V. Aristov, V. Fradkov, L. Shvindlerman, Detachment of the migrating grain-boundary half-loop from an adsorbed impurity cloud, Soviet Physics - Solid State 22 (1980) 1055-9.

[63] K. Janssens, D. Olmsted, E. Holm, S. M. Foiles, S. Plimpton, P. Derlet, Computing the mobility of grain boundaries., Nature Mater. 5 (2006) 124-127.

[64] D. Olmsted, E. Holm, S. M. Foiles, Survey of computed grain boundary properties in face-centered cubic metals - ii: Grain boundary mobility, Acta Mater. 57 (2009) 3704-3713.

[65] H. Zhang, D. Srolovitz, J. Douglas, J. Warren, Atomic motion during the migration of general [001] tilt grain boundaries in Ni, Acta Mater. 55 (2007) 4527-4533. 
[66] J. Hoshen, R. Kopelman, Percolation and cluster distribution. i. cluster multiple labeling technique and critical concentration algorithm, Phys. Rev. B 14 (1976) 3438-3445.

[67] D. Fan, L.-Q. Chen, Computer simulation of grain growth using a continuum field model, Acta Mater. 45 (1997) 611-622. 
Recent experimental and theoretical findings suggest that nanocrystalline binary alloys can be stabilized against interface-driven homogenization processes via grain boundary (GB) solute segregation mechanism. However, a detailed understanding of this process requires detangling the thermodynamic aspect, GB energy, from the kinetic one, GB mobility. In this work, we present a diffuse-interface model of GB segregation in binary metallic alloys that is capable of accounting for bulk thermodynamics, interfacial energies, and the interaction of alloying elements with GBs. In addition, the model presented herein extends current treatments by independently treating solute-solute interactions within both the bulk grain and GB regions, allowing for deviations from dilute and ideal systems and the ability to account for phase separation processes occurring in conjunction with grain growth. Starting with the analytical treatment of onedimensional (1D) systems, we investigate the dependence of the GB energy, and subsequently the driving force for grain growth, on the segregation model parameters. More specifically, classic GB segregation isotherms are recovered in the limit of 1D infinite grains. Simulation results of two-dimensional systems reveal regimes of increased thermal stability, and highlight the importance of the thermodynamic model parameters of both bulk grain and GBs on grain growth processes. In broader terms, our modeling approach provides further avenues to explore GB solute segregation and its role in stabilizing polycrystalline aggregates. 